\title{
NMR and NBO calculation of benzimidazoles and pyrimidines: Nano physical parameters investigation
}

\author{
M. Monajjemi ${ }^{1 \star}$, M. Sheikhi ${ }^{2}$, M. Mahmodi Hashemi ${ }^{1}$, F. Molaamin ${ }^{3}$ and R. Zhiani ${ }^{1}$ \\ ${ }^{1}$ Department of Chemistry, Science and Research Branch, Islamic Azad University, Tehran, Iran. \\ ${ }^{2}$ Ph.D Student, Science and Research Branch, Islamic Azad University, Tehran, Iran. \\ ${ }^{3}$ Department of Chemistry, Qom Branch, Islamic Azad University, Qom, Iran.
}

Accepted 2 June, 2011

\begin{abstract}
Six theoretical methods were used for the calculation of physical parameters for derivatives of pyrimidines and benzimidazols. We used Gaussian 98 at NMR and NBO calculations by using HF method with 6-31G, 6-31G* and 6-31+G basis set and B3LYP, BLYP and B3PW91 methods with 6-31G basis set. Chemical shift was drawn curve for all of the atoms in each molecule. The thermo chemical parameters including thermal energy $(\Delta E)$, atomic charges, chemical shift anisotropy( $\delta)$, asymmetry parameter $(\eta)$, chemical shift anisotropy $(\Delta \sigma)$, dipole orientation, isotropic, anisotropic, NMR determinant and distance matrix compounds. Also the natural bond orbital (NBO) analysis has been performed which seem informative to show some important atomic and structural features.
\end{abstract}

Key words: Benzimidazoles, pyrimidines, NBO analysis, Nano physical parameters.

\section{INTRODUCTION}

Recently, medicinal chemists have taken great interest in the combinatorial methods for the design and synthesis of pharmacologically-relevant heterocyclic molecules (Dolle and Nelson, 1999; Dolle, 2000, 2004). Inexpensive and readily-available domestic microwave ovens transform electromagnetic energy into heat, thus the absorption and transmission of the energy varies greatly from that of conventional heating. The benzimidazole nucleus is an important structure in numerous natural and synthetic compounds and in medicinal chemistry. Solidphase approaches for benzimidazole synthesis have been published (Smith et al., 1999), however the cyclization of benzimidazole catalyzed by aluminamethanesulfonic acid (AMA) under microwave irradiation has not been reported. The appropriate carboxylic acids were reacted with 1,2 phenylenediamine in the presence of AMA under microwave irradiation to give the corresponding benzimidazoles in good to excellent yields (Scheme 1).

Structures containing benzimidazole, well-known to have a wide range of biological properties, have commercial applications in various realms of therapy, including

\footnotetext{
*Corresponding author. E-mail: m_monajjemi@yahoo.com
}

antiulcerative, anti-hypertensive, antiviral, antifungal, antitumor and antihistaminic agents, and antihelminthic agents in veterinary medicine (Spasov et al., 1999). Benzimidazole derivatives have found the appreciation in diverse therapeutic areas including antimicrobial activity (Kus et al., 2004; Wu et al., 2003; Mollaamin et al., 2008; Ozden et al., 2005; Sztanke et al., 2006), the activity against several viruses such as HIV (Porcari et al ., 1998; Mollaamin et al., 2010; Samia et al., 2006), antiallergic (Kilcig and Altanlar, 2006; Nakano et al., 1999), antioxidant, antihistaminic (Vijayakumar and Jafar Ahamed, 2010), antitubercular (Yadav and Srivastava, 2011; Kuchkguzel et al., 2001), antiasthmatic (Souness et al., 2000), antidiabetic (Senten et al., 2003; Black et al., 2005), anticancer (Pal et al., 2011; Sun et al., 2011; Kruse et al.,1989; Islam et al.,1991; Ramla et al.,2007), antitumor (Denny et al., 1990 ; Tatsuta et al., 2005), antiulcer (Jung et al., 1993; Hazelton et al., 1995), antihelmentic (Karen, 2006), HIV-1 reverse transcriptase inhibitors (Gardiner et al., 2003), anticoagulant (Young et al., 2006), anti inflammatory(Fox et al., 2009), antibacterial (Rosowsky et al., 1997; Andrzejewska et al., 2004), the series of biologically active benzimidazoles (AJafar et al.,2009). Furthermore, these heterocycles are considered to be privileged structures by medicinal chemists. 

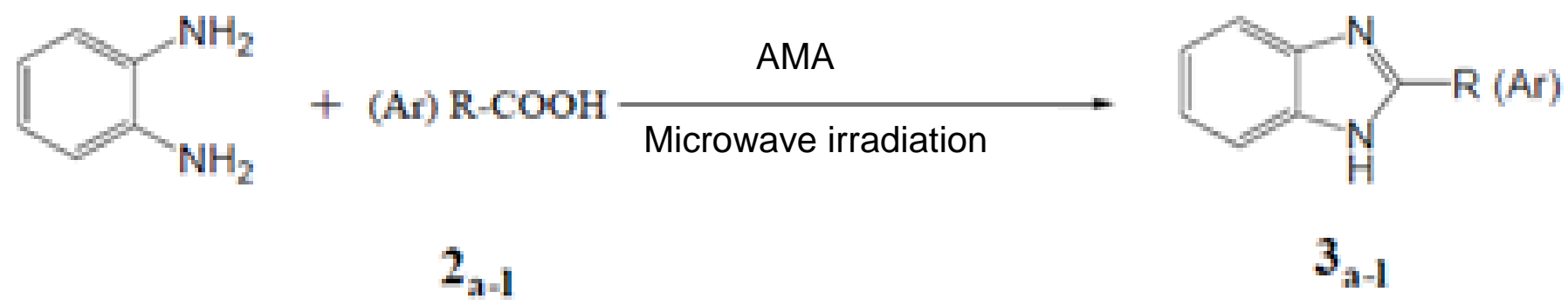

$3_{\mathrm{a}-1}$

Scheme 1. Carboxylic acids reaction with 1, 2 phenylenediamine in the presence of AMA under microwave irradiation to give the corresponding benzimidazoles in good to excellent yields.<smiles>CCOC(=O)CC(C(=O)OCC)C(=O)OCC(C)=O</smiles>

Scheme 2. Preparation of dihydropyrimidinones through Biginelli reaction under mild conditions.

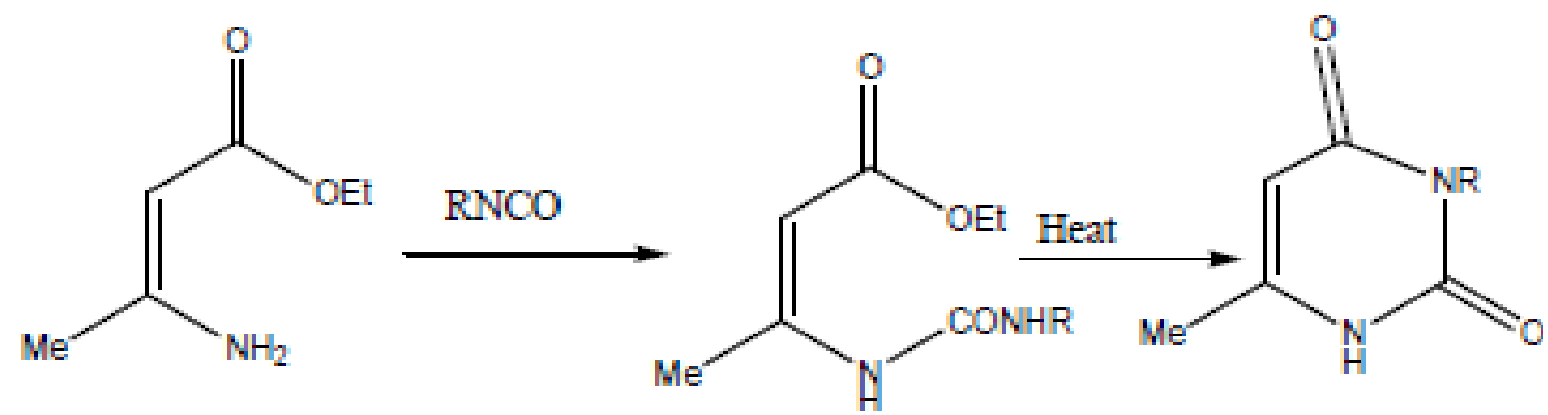<smiles>COC(=O)C1=C(C)NC(=O)NC1c1ccc(C)o1</smiles>

A

Scheme 3. Ethyl $\beta$ - aminocrotonate undergoes an extremely facile reaction with phenylisocyanate or methyl isocyanate to form an intermediate ureido derivative which undergoes cyclization.

This seems to be because pyrimidines represents one of the most active class of compounds possessing wide spectrum of biological activity, namely, significant in vitro activity against unrelated DNA and RNA, viruses including polio herpes viruses, diuretic, antitumor, anti HIV, cardiovascular (Kappe,1993). Biginelli compounds show a diverse range of biological activities. The interest focused on Biginelli compounds leading to the development of Nitractin (A) (Marsault and Peterson, 2011), that has excellent activity against the virus of trachoma group, the same compounds also exhibit antibacterial activity. Application of dodecylphosphonic acid (DPA) (Ghassamipour and Sardarian, 2009), solid Bronsted acid, is reported for the preparation of dihydro-pyrimidinones through Biginelli reaction under mild conditions (Scheme 2).

Ethyl $\beta$ - aminocrotonate undergoes an extremely facile reaction with phenylisocyanate or methyl isocyanate to form an intermediate ureido derivative which undergo cyclization as treatment with base (Ghassamipour and Sardarian, 2009; Gupta et al., 2010) (Scheme 3).

\section{METHODS OF COMPUTATION}

In Stage 1, we started a ChemDraw and constructed molecules, after which the results were saved as a ChemDraw file. In Stage 2, this file was reopened using Chem3D and an energy minimization was performed. Then, the results were saved as a gjc file. In Stage 3 , this file was reopened using Gaussian98 and the calculations were performed using the Gaussian $\AA 98$ program suite. Gaussian is one of the most widely used quantum chemical program packages for molecular applications, and is used both in industry and in many scientific areas in academia. we have calculated the geometric parameters of the compounds in the ground state using 
the Hartree-Fock (HF) (Moller and Plesset, 1934), Becke's three parameter hybrid method (Becke, 1988) with the Lee et al. (1988) correlation functional methods (B3LYP), Becke's exchange functional in combination with the Lee, Yang and Parr correlation functional methods (BLYP) (Lee et al., 1988; Becke, 1993), Becke's three parameter exchange functional combined with gradient corrected correlation functional of Perdew and Wang's 1991 (B3PW91) (Becke,1993; Predew and Wang,1992), and 6-31G, 6-31G* and 6$31+\mathrm{G}$ basis set.

The calculation that one asks Gaussian to perform is distributed between many processors to get the answer faster. If one wants to optimize a geometry, it means that one wants Gaussian to adjust the bond lengths, angles and dihedrals to find the lowest energy conformation of the molecule. The command used to tell Gaussian to optimize the molecular geometry is "opt".

The Gaussian program does semi-empirical and ab initio calculations. In $a b$ initio calculations the important integrals are done directly from first principles. First principles means that the integrals are done either using closed formulas or by doing the integrals numerically. Particularly, ab initio method works best for calculating NMR properties (Monaijemi et al., 2010; Monajjemi et al., 2007). Finding a good geometry is called geometry optimization, so "OPT" are used as the keyword.

The calculation will generate an output file called "filename. out". The output file (filename.out) contains a lot of information about the calculation and the results. The content depends on what type of calculation that has been performed and on what print options that was specified. The units are usually Hartree (atomic unit) for energy and Angstrom for distance. There are several different pieces of data that you may need from this. The important information are the Hartree-Fock energy $(\Delta \mathrm{E})$, the Mulliken charges, Distance matrix (angstroms), Dipole moment (Debye) and Atomic charge. Distance matrix value is determined (Monajjemi et al., 2008; Mollaamin et al., 2011; Monajjemi et al., 2003).

We used Gaussian 98 at the NMR shift calculation using the HF, B3LYP, BLYP, B3PW91 methods and 6-31G, 6-31G* and 6$31+G$ basis set. There for "NMR" are used as the keyword (Monaijemi et al., 2003). The calculation will generate an output file called "NMR.out". The output file (NMR.out) contains a lot of information about the NMR shift calculation and are listed in the "GIAO Magnetic shielding tensor (ppm)", such as $\sigma$ isotropic (ppm) and $\sigma$ anisotropic (ppm). As the usig the $X, Y$ and $z$ coordinates determinant $3^{*} 3$ is solved and $\sigma$ is calculated. Molecular orbital calculations can be used to get good estimates for chemical shifts. In this exercise we calculated the chemical shifts for each of the atom.

In the part of "GIAO magnetic shielding tensor (ppm)" the using the $\sigma$ Isotropic (ppm), $\sigma$ anisotropic (ppm) and Eigen values $\left(\sigma_{11}\right.$, $\left.\sigma_{22}, \sigma_{33}\right)$ are calculated parameters such as $\delta, \eta$ and $\Delta \sigma$ (Monajjemi et al., 2006).

We obtained Shielding value for the each atom with Gauss View program (GIAO Magnetic shielding).

A full NBO analysis is obtained in Gaussian when using the $\mathrm{POP}=\mathrm{NBO}$ keyword. NBO analysis did using the HF, B3LYP, BLYP, B3PW91 methods and $6-31 \mathrm{G}, 6-31 \mathrm{G}^{*}$ and $6-31+\mathrm{G}$ basis set and the output is obtained for each of the molecule (Monajjemi et al., 2006). The main listing of NBOs, displaying the form and occupancy of the complete set of NBOs that span the input AO space and for each orbital gives the type of orbital and the occupancy. We were extracted just BD for 2-center bond and BD* for 2center antibond from "NBO.output". For example at the Molecule 1.

\section{RESULTS AND DISCUSSION}

In this work, we calculated parameters like atomic charges, energy $(\Delta \mathrm{E})$, chemical shift anisotropy $(\delta$, asym- metry parameter $(\eta)$, chemical shift anisotropy $(\Delta \sigma)$, dipole orientation, isotropic, anisotropic, NMR determinant and distance matrix determinant for several benzimidazoles and pyrimidines using the HF method with 6-31G,6-31G* and $6-31+G$ basis set and B3LYP, BLYP and B3PW91 methods with 6-31G basis set. These parameters are shown in Tables 1 and 2. At present, we considered these parameters.

As shown in Table 1, in Molecule 1, at HF/6-31G, HF/6$31 \mathrm{G}^{*}$ and B3PW91/6-31G, $\mathrm{N}_{1}, \mathrm{~N}_{3}, \mathrm{C}_{5}$ atoms contain negative atomic charge values, while in the other levels just $\mathrm{N}_{1}$ and $\mathrm{N}_{3}$ atoms contain negative atomic charge values. In Molecule $1 \mathrm{C}_{2}$ atom for all methods has the positive value. In Molecule 2, in all of the methods, except $\mathrm{BLYP} / 6-31 \mathrm{G}$ level, $\mathrm{N}_{3}$ and $\mathrm{N}_{5}$ atoms have the greatest negative atomic charge values and $\mathrm{C}_{4}$ has the greatest positive amount. In Molecule 3 in all of the methods, $\mathrm{N}_{3}$ and $\mathrm{N}_{5}$ atoms have the greatest negative atomic charge values, and $\mathrm{C}_{4}$ atom has the greatest positive atomic charge amount. In Molecule 4 , at all of the methods, $N_{3}$ and $\mathrm{N}_{5}$ have the greatest negative atomic charge, also at all of the methods except HF/6-31+G level $C_{1}, N_{3}$ and $N_{5}$ atoms have negative values. In Molecule $5, N_{5}$ has the greatest negative atomic charge. Also at $\mathrm{HF} / 6-31+\mathrm{G}, \mathrm{C}_{6}$ and $\mathrm{C}_{9}$ have negative Atomic charge, while in the other levels $\mathrm{C}_{7}$ and $\mathrm{C}_{10}$ have positive atomic charge.

As pointed in Table 1, for Molecule 1, in all of the methods used, except HF/6-31G* chemical shift anisotropy $(\delta), C_{6}$ has greatest negative value and $C_{9}$ is greatest positive value, but at $\mathrm{HF} / 6-31 \mathrm{G}^{*}, \mathrm{C}_{6}$ is greatest negative value and $\mathrm{C}_{4}$ is greatest positive value. For Molecule 2 at all methods, except BLYP, $\mathrm{C}_{7}$ has the greatest positive amount. In HF/6-31G, $\mathrm{C}_{4}$ has greatest negative amount, while at $\mathrm{HF} / 6-31 \mathrm{G}^{*}$ and $\mathrm{HF} / 6-31+\mathrm{G}, \mathrm{C}_{2}$ has greatest negative amount and at B3LYP and B3PW91 methods, $\mathrm{C}_{4}$ has greatest positive amount. As shown in Molecule 2 for BLYP method has not response. For Molecule 3 in all methods, $C_{7}$ has the greatest positive amount. As shown at HF/6-31G, HF/6-31G* and $\mathrm{HF} / 6-31+\mathrm{G}, \mathrm{C}_{2}$ has greatest negative amount and in B3LYP, BLYP and B3PW91 methods, $\mathrm{C}_{4}$ has greatest negative amount, also in BLYP method, $\mathrm{C}_{2}$ has greatest positive amount. For Molecule 4 at all methods, $N_{5}$ has the greatest positive amount and $\mathrm{C}_{4}$ has greatest negative amount except BLYP method that $\mathrm{N}_{3}$ has greatest negative amount. For Molecule 5 at all methods, $\mathrm{C}_{4}$ has the greatest negative amount and $N_{5}$ has greatest positive amount.

As pointed in Table 1, in Molecule 1, in all of the methods except BLYP, for the atoms is shown in Table 1, $\eta$ has the positive amounts. In the BLYP method, $N_{3}$ has the negative amount. In Molecule 2, at all of the methods $\eta$ has positive amounts. Asymmetry parameter $(\eta)$ value in $\mathrm{HF} / 6-31 \mathrm{G}, \mathrm{HF} / 6-31 \mathrm{G}^{*}$, B3PLY/6-31G and B3PW91/6$31 \mathrm{G}$ levels for the $C_{1}$ and $N_{5}$ atoms, also in $\mathrm{HF} / 6-31+\mathrm{G}$ level for the $\mathrm{C}_{4}$ and $\mathrm{N}_{5}$ atoms is the greatest value. In Molecule 3 , at all of the methods used for the atoms are shown in Table 1, $\eta$ has the positive amounts. The $\eta$ value in all of 
Table 1. Values of parameters like atomic charges, $\Delta \mathrm{E}(\mathrm{kcal} / \mathrm{mol})$, chemical shift $(\delta)$, asymmetry parameter $(\eta)$, chemical shift anisotropy $(\Delta \sigma)$, dipole moment for active site of studied moleculars obtained using different methods.

\begin{tabular}{|c|c|c|c|c|c|c|c|c|c|c|c|c|c|c|c|c|c|c|c|c|c|c|c|c|c|c|c|c|c|c|c|c|c|c|c|c|c|}
\hline \multirow{2}{*}{\multicolumn{2}{|c|}{\begin{tabular}{|l} 
Method \\
Basis st
\end{tabular}}} & \multicolumn{18}{|c|}{ HF } & \multicolumn{6}{|c|}{ B3LYP } & \multicolumn{5}{|c|}{ BLYP } & \multicolumn{7}{|c|}{ B3PW91 } \\
\hline & & \multicolumn{6}{|c|}{$6-31 G$} & \multicolumn{6}{|c|}{ 6-31G* } & \multicolumn{6}{|c|}{$6-31+G$} & \multicolumn{18}{|c|}{$6-31 G$} \\
\hline 竞 & $\mid$ & 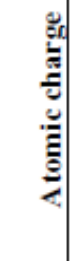 & 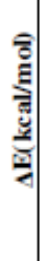 & $\delta$ & $\eta$ & $\Delta \sigma$ & 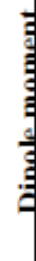 & 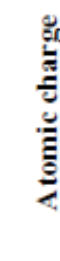 & 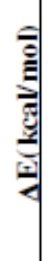 & $\delta$ & $\eta$ & $\Delta \boldsymbol{\sigma}$ & 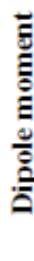 & 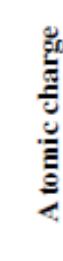 & $\frac{\bar{g}}{\bar{s}}$ & 8 & $\eta$ & $\Delta \sigma$ & 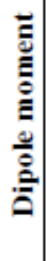 & 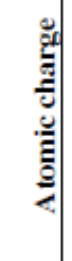 & 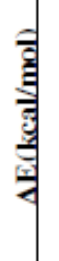 & $\delta$ & $\eta$ & $\Delta \boldsymbol{\sigma}$ & 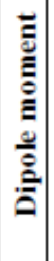 & 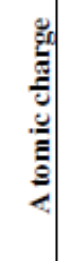 & 氧 & $\delta$ & $\eta$ & $\Delta \sigma$ & 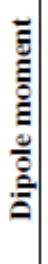 & 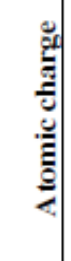 & 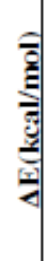 & $\delta$ & $\eta$ & $\Delta \sigma$ & 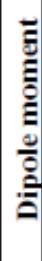 \\
\hline $\begin{array}{l}\overline{0} \\
\bar{z} \\
\frac{3}{0} \\
z\end{array}$ & $\begin{array}{l}N(1) \\
C(2) \\
N(3) \\
C(4) \\
C(5) \\
C(5) \\
C(9)\end{array}$ & $\begin{array}{l}-1.0 \\
1.07 \\
-1.03 \\
0.51 \\
-0.31 \\
0.04 \\
0.5\end{array}$ & 。 & $\begin{array}{r}-6932 \\
-11023 \\
-7974 \\
13291 \\
7.73 \\
-118279 \\
141800\end{array}$ & $\begin{array}{l}0.35 \\
0.41 \\
0.04 \\
0.76 \\
0.75 \\
0.63 \\
0.58\end{array}$ & $\begin{array}{c}-104 \\
-165.34 \\
.120 .06 \\
199.37 \\
116.50 \\
178.18 \\
212.40\end{array}$ & $\stackrel{్}{\sigma}$ & $\begin{array}{l}.992 \\
1.04 \\
0.91 \\
0.46 \\
.0 .37 \\
0.5 \\
0.55\end{array}$ & 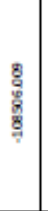 & $\begin{array}{l}.5101 \\
-.875 \\
.6500 \\
12.656 \\
6753 \\
.95 .92 \\
115.51 \\
\end{array}$ & $\begin{array}{l}0.13 \\
0.32 \\
0.23 \\
0.62 \\
0.87 \\
0.50 \\
0.54\end{array}$ & 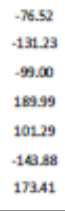 & हू & $\begin{array}{l}-0.95 \\
0.97 \\
-0.91 \\
0.72 \\
0.95 \\
0.51 \\
0.25\end{array}$ & ఫูँ & $\begin{array}{r}-66 \\
-10 \\
-80 \\
13 \\
7 \\
-11 \\
36 .\end{array}$ & $\begin{array}{l}0.32 \\
0.44 \\
0.08 \\
0.76 \\
0.78 \\
0.54 \\
0.57\end{array}$ & $\begin{array}{l}.99 \pi \\
-16190 \\
.120 .72 \\
198.46 \\
115.5 \\
.174 .83 \\
.2139\end{array}$ & 思 & $\begin{array}{l}-0.70 \\
0.69 \\
-0.73 \\
0.25 \\
0.003 \\
0.5 \\
0.28\end{array}$ & $\begin{array}{l}\frac{a}{2} \\
\frac{d}{d y}\end{array}$ & $\begin{array}{l}-73.18 \\
.9621 \\
-72.96 \\
109.94 \\
76.01 \\
-101.34 \\
119.05\end{array}$ & $\begin{array}{l}0.23 \\
0.12 \\
0.15 \\
0.58 \\
0.47 \\
0.39 \\
0.55\end{array}$ & $\begin{array}{l}-109.77 \\
-141.32 \\
-112.44 \\
164.91 \\
162.18 \\
-152.01 \\
178.58\end{array}$ & बृत् & $\begin{array}{l}-0.51 \\
0.50 \\
-0.54 \\
0.31 \\
0.07 \\
0.41 \\
0.23\end{array}$ & 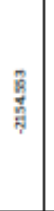 & $\begin{array}{l}-75.14 \\
-89.11 \\
-78.23 \\
1027 \\
73.58 \\
-96.23 \\
11083\end{array}$ & $\begin{array}{c}0.16 \\
4.25 \\
.78 .23 \\
0.52 \\
0.41 \\
0.26 \\
0.6\end{array}$ & $\begin{array}{l}-11272 \\
-13367 \\
.117 .35 \\
154.19 \\
110.37 \\
-16435 \\
166.25\end{array}$ & बू. & $\begin{array}{l}-0.76 \\
0.74 \\
-0.78 \\
0.37 \\
-0.04 \\
0.53 \\
0.3\end{array}$ & 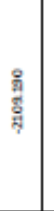 & $\begin{array}{l}-72.80 \\
-93.09 \\
-78.78 \\
109.52 \\
75.87 \\
.100 .21 \\
118.84\end{array}$ & $\begin{array}{l}0.30 \\
0.12 \\
0.05 \\
0.58 \\
0.45 \\
0.99 \\
0.53\end{array}$ & $\begin{array}{l}-209.20 \\
-139.63 \\
-118.17 \\
164.28 \\
113.81 \\
-150.32 \\
178.27\end{array}$ & 超 \\
\hline 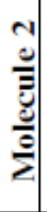 & $\begin{array}{l}c(1) \\
c(1) \\
N(3) \\
c(4) \\
N(5) \\
c(\beta) \\
c(7)\end{array}$ & $\begin{array}{l}-0.10 \\
0.20 \\
-0.98 \\
1.05 \\
-0.93 \\
0.01 \\
-0.03\end{array}$ & 。 & $\begin{array}{r}-97.01 \\
-102.42 \\
-2060 \\
-10317 \\
-7118 \\
21.86 \\
130.40\end{array}$ & $\begin{array}{l}0.74 \\
0.56 \\
0.16 \\
0.69 \\
0.99 \\
0.03 \\
0.53\end{array}$ & $\begin{array}{l}-14551 \\
.15963 \\
.10591 \\
-154.76 \\
.10078 \\
32.79 \\
195.73\end{array}$ & 象 & $\begin{array}{l}.099 \\
0.11 \\
0.86 \\
100 \\
.094 \\
.0 .06 \\
0.08\end{array}$ & $\frac{\pi}{2}$ & $\begin{array}{c}-69.76 \\
-9.7 .76 \\
-55.34 \\
-62.72 \\
69.81 \\
20.50 \\
126.16 \\
19.16\end{array}$ & $\begin{array}{l}0.72 \\
0.57 \\
0.29 \\
0.59 \\
0.70 \\
0.30 \\
0.51\end{array}$ & 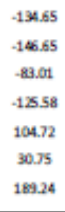 & $\frac{1}{2}$ & $\begin{array}{l}0.36 \\
.0 .18 \\
-0.06 \\
0.92 \\
.0 .28 \\
.0 .65 \\
0.49\end{array}$ & के & $\begin{array}{l}.98 \\
-10 \\
-70 \\
-10 \\
.76 \\
2 . \\
13 .\end{array}$ & $\begin{array}{l}0.74 \\
0.56 \\
0.21 \\
0.75 \\
0.95 \\
0.02 \\
0.54\end{array}$ & $\begin{array}{l}-148.14 \\
-152.80 \\
.406 .02 \\
-152.21 \\
-111.19 \\
34.25 \\
196.70\end{array}$ & $\frac{1}{8}$ & $\begin{array}{l}0.01 \\
0.010 \\
-0.70 \\
0.67 \\
-0.66 \\
-0.10 \\
0.06\end{array}$ & 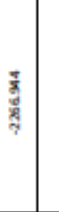 & $\begin{array}{l}-76.32 \\
.82 .11 \\
-67.82 \\
-91.73 \\
0.60 \\
22.51 \\
11.0 .02\end{array}$ & $\begin{array}{l}0.97 \\
0.72 \\
0.11 \\
0.35 \\
0.88 \\
0.17 \\
0.00\end{array}$ & $\begin{array}{l}-134.48 \\
-123.16 \\
-102.74 \\
-139.50 \\
10591 \\
33.7 \\
178.54\end{array}$ & 奋 & : & 旅 & : & : & . & 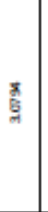 & $\begin{array}{l}-0.00 \\
0.10 \\
-0.75 \\
0.71 \\
-0.00 \\
-0.13 \\
0.03\end{array}$ & $\begin{array}{l}\text { है } \\
\text { से }\end{array}$ & $\begin{array}{l}-75.95 \\
-81.70 \\
-68.02 \\
-99.55 \\
70.98 \\
21.90 \\
118.40\end{array}$ & $\begin{array}{l}0.97 \\
0.73 \\
0.99 \\
0.36 \\
0.90 \\
0.20 \\
0.39\end{array}$ & $\begin{array}{l}-113.92 \\
-122.55 \\
.20203 \\
-125.82 \\
106.47 \\
32.85 \\
17.50\end{array}$ & $\frac{2}{3}$ \\
\hline 窇 & $\begin{array}{l}c(1) \\
c(2) \\
N(3) \\
c(4) \\
N(5) \\
c(b) \\
c(1)\end{array}$ & $\begin{array}{l}-0.22 \\
0.24 \\
-0.98 \\
1.05 \\
-0.93 \\
0.03 \\
0.32\end{array}$ & $\circ$ & $\begin{array}{c}87.45 \\
-113.48 \\
-6656 \\
-107.23 \\
.7932 \\
19.70 \\
122 n\end{array}$ & $\begin{array}{l}0.78 \\
0.83 \\
0.14 \\
0.56 \\
0.80 \\
0.34 \\
0.90\end{array}$ & $\begin{array}{l}131.18 \\
-170.22 \\
.99 .84 \\
180.05 \\
.118 .99 \\
29.55 \\
184.08\end{array}$ & 常 & $\begin{array}{l}0.22 \\
0.06 \\
0.87 \\
1.00 \\
0.86 \\
0.02 \\
0.39\end{array}$ & 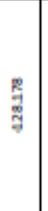 & 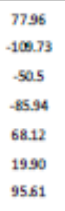 & $\begin{array}{l}0.82 \\
0.81 \\
0.02 \\
0.47 \\
0.88 \\
0.22 \\
0.97\end{array}$ & $\begin{array}{l}116.95 \\
-168.50 \\
-75.79 \\
-128.92 \\
102.18 \\
29.94 \\
143.42\end{array}$ & 8 & $\begin{array}{l}0.81 \\
.0 .54 \\
-0.86 \\
0.93 \\
.0 .83 \\
.009 \\
.0 .09\end{array}$ & $\frac{8}{7}$ & $\begin{array}{c}88 \\
-11 \\
-60 \\
-10 \\
86 \\
19 \\
12\end{array}$ & $\begin{array}{l}0.73 \\
0.85 \\
0.10 \\
0.51 \\
0.77 \\
0.96 \\
0.99\end{array}$ & $\begin{array}{l}13230 \\
-128.26 \\
.92 .47 \\
.158 .43 \\
.121 .09 \\
29.89 \\
183.59\end{array}$ & 8 & $\begin{array}{l}0.01 \\
0.13 \\
-0.69 \\
0.68 \\
-0.66 \\
-0.08 \\
0.15\end{array}$ & ్ֶ. & $\begin{array}{l}81.65 \\
29.71 \\
.6404 \\
.9672 \\
-7258 \\
22.63 \\
97.25\end{array}$ & $\begin{array}{l}0.51 \\
0.97 \\
0.17 \\
0.24 \\
0.90 \\
0.17 \\
0.95\end{array}$ & $\begin{array}{l}12248 \\
13457 \\
.9606 \\
-12.08 \\
.10 .88 \\
33.95 \\
14587\end{array}$ & $\frac{\pi}{2}$ & $\begin{array}{l}0.08 \\
0.10 \\
-0.50 \\
0.50 \\
-0.58 \\
-0.09 \\
0.11\end{array}$ & 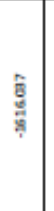 & $\begin{array}{l}7899 \\
84.10 \\
-62.37 \\
.90 .63 \\
6898 \\
24.56 \\
89.70\end{array}$ & $\begin{array}{l}0.48 \\
0.92 \\
0.15 \\
0.11 \\
0.50 \\
0.05 \\
0.95\end{array}$ & $\begin{array}{l}118.48 \\
126.15 \\
.9355 \\
.13595 \\
.10347 \\
3684 \\
.134 .56\end{array}$ & है & $\begin{array}{l}-0.03 \\
0.13 \\
-2.74 \\
0.72 \\
-0.00 \\
-0.11 \\
0.15\end{array}$ & 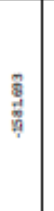 & $\begin{array}{l}81.38 \\
89.91 \\
-63.79 \\
.93 .68 \\
.73 .56 \\
21.83 \\
98.06\end{array}$ & $\begin{array}{l}0.51 \\
0.96 \\
0.23 \\
0.24 \\
0.76 \\
0.21 \\
0.90\end{array}$ & $\begin{array}{l}12.07 \\
13.48 \\
.95 .69 \\
-120.22 \\
-110.34 \\
32.75 \\
127.08\end{array}$ & 苗 \\
\hline 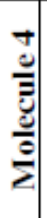 & $\begin{array}{l}c(1) \\
c(1) \\
N(3) \\
N(10) \\
c(4) \\
N(9) \\
N(1)\end{array}$ & $\begin{array}{l}0.0 .44 \\
0.34 \\
-0.99 \\
0.39 \\
0.37 \\
-0.53 \\
0.23\end{array}$ & $\circ$ & $\begin{array}{c}103.9 \\
107.08 \\
-76.23 \\
7.22 \\
.8990 \\
27.23 \\
2.84\end{array}$ & $\begin{array}{l}0.38 \\
0.68 \\
0.62 \\
0.74 \\
0.65 \\
0.71 \\
1.00\end{array}$ & $\begin{array}{c}155.79 \\
1.00 .54 \\
.11635 \\
11.74 \\
.14234 \\
415.25 \\
4.27\end{array}$ & 晜 & $\begin{array}{l}0.2 \\
0.28 \\
0.83 \\
0.07 \\
0.00 \\
0.56 \\
0.2\end{array}$ & 峯 & $\begin{array}{l}10129 \\
10258 \\
.71 .40 \\
-7.89 \\
-8794 \\
24957 \\
-4.49\end{array}$ & $\begin{array}{l}0.39 \\
0.66 \\
0.37 \\
0.55 \\
0.69 \\
0.57 \\
0.59\end{array}$ & $\begin{array}{l}15193 \\
154.02 \\
-197.11 \\
-11.94 \\
-131.91 \\
374.36 \\
-674\end{array}$ & ดू & $\begin{array}{l}-0.16 \\
0.76 \\
-0.93 \\
0.48 \\
0.65 \\
-0.36 \\
0.25\end{array}$ & बุ & 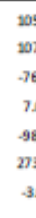 & $\begin{array}{l}0.37 \\
0.57 \\
0.39 \\
0.84 \\
0.59 \\
0.71 \\
0.85\end{array}$ & $\begin{array}{l}15798 \\
16150 \\
-414.17 \\
10.58 \\
.427 .69 \\
41078 \\
.661\end{array}$ & 最 & $\begin{array}{l}0.04 \\
0.32 \\
-0.75 \\
0.32 \\
0.25 \\
-0.05 \\
0.16\end{array}$ & 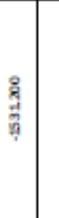 & $\begin{array}{l}91.17 \\
92.96 \\
.7482 \\
6.15 \\
.7867 \\
252.92 \\
.290\end{array}$ & $\begin{array}{l}0.30 \\
0.59 \\
0.41 \\
0.85 \\
0.50 \\
0.71 \\
0.81\end{array}$ & $\begin{array}{l}136.76 \\
13965 \\
.12 .23 \\
9.28 \\
.138 .00 \\
37938 \\
.436\end{array}$ & 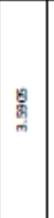 & $\begin{array}{l}0.05 \\
0.31 \\
-0.57 \\
0.29 \\
0.23 \\
-0.36 \\
0.14\end{array}$ & $\frac{5}{\frac{3}{3}}$ & $\begin{array}{c}85.85 \\
8857 \\
.74 .14 \\
5.64 \\
.73 .96 \\
242.174 \\
.282\end{array}$ & $\begin{array}{l}0.28 \\
0.58 \\
0.36 \\
0.23 \\
0.54 \\
0.71 \\
0.73\end{array}$ & $\begin{array}{c}130.27 \\
132.85 \\
-110.28 \\
8.46 \\
-11079 \\
36326 \\
4.23\end{array}$ & 글 & $\begin{array}{l}0.02 \\
0.32 \\
.0 .80 \\
0.34 \\
0.25 \\
.0 .2 \\
0.20\end{array}$ & 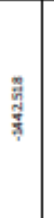 & $\begin{array}{l}90.12 \\
91.88 \\
-74.13 \\
6.44 \\
-77.59 \\
252.02 \\
-2.99\end{array}$ & $\begin{array}{l}0.30 \\
0.50 \\
0.45 \\
0.83 \\
0.51 \\
0.71 \\
0.91\end{array}$ & $\begin{array}{c}135.18 \\
137.53 \\
-112.20 \\
9.67 \\
-116.38 \\
372.03 \\
4.49\end{array}$ & 音 \\
\hline 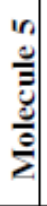 & $\begin{array}{l}c(1) \\
c(2) \\
c(4) \\
N 55) \\
c(1) \\
c(9) \\
c(17)\end{array}$ & $\begin{array}{l}0.03 \\
0.28 \\
0.37 \\
-0.50 \\
0.50 \\
0.54 \\
0.22\end{array}$ & - & $\begin{array}{c}91.52 \\
84.84 \\
.90 .67 \\
298.63 \\
132.02 \\
136.22 \\
5.7\end{array}$ & $\begin{array}{l}0.57 \\
0.78 \\
0.95 \\
0.82 \\
0.78 \\
0.58 \\
0.65\end{array}$ & $\begin{array}{c}137.28 \\
127.26 \\
-136.01 \\
447.94 \\
198.18 \\
204.93 \\
8.65\end{array}$ & है & $\begin{array}{l}0.0 \\
0.18 \\
0.0 \\
.0 .52 \\
0.54 \\
0.56 \\
0.25\end{array}$ & 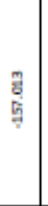 & $\begin{array}{c}87 . A 2 \\
81.01 \\
-8.197 \\
26987 \\
10621 \\
110.088 \\
5.14\end{array}$ & $\begin{array}{l}0.65 \\
0.64 \\
0.87 \\
0.67 \\
0.75 \\
0.65 \\
0.69\end{array}$ & $\begin{array}{l}131.13 \\
121.51 \\
-12.06 \\
404.81 \\
159.32 \\
165.13 \\
7.7159\end{array}$ & 8 & $\begin{array}{l}0.03 \\
0.60 \\
0.52 \\
.0 .32 \\
-.0 .03 \\
.0 .08 \\
0.25\end{array}$ & $\frac{?}{8}$ & $\begin{array}{l}93 \\
85 \\
.96 \\
29 \\
13 . \\
13 \\
5 .\end{array}$ & $\begin{array}{l}0.56 \\
0.75 \\
0.88 \\
0.81 \\
0.75 \\
0.55 \\
0.57\end{array}$ & $\begin{array}{c}139.54 \\
128.63 \\
-13.37 \\
445.13 \\
19991 \\
205.65 \\
8.49\end{array}$ & $\underset{m}{5}$ & $\begin{array}{c}0.06 \\
0.31 \\
0.7 \\
-0.37 \\
0.31 \\
0.33 \\
0.17\end{array}$ & 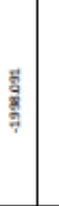 & $\begin{array}{c}79.55 \\
7.34 \\
-72.59 \\
277.29 \\
112.38 \\
11.28 \\
4.37\end{array}$ & $\begin{array}{l}0.54 \\
0.53 \\
0.79 \\
0.86 \\
0.56 \\
0.51 \\
0.80\end{array}$ & $\begin{array}{c}11978 \\
116.51 \\
-10.38 \\
41593 \\
168.57 \\
172.25 \\
6.56\end{array}$ & 糔 & $\begin{array}{l}0.07 \\
0.31 \\
0.25 \\
-0.34 \\
0.27 \\
0.28 \\
0.15\end{array}$ & 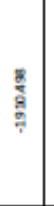 & $\begin{array}{c}75.38 \\
75.60 \\
-69.61 \\
2662 \\
10500 \\
10697 \\
3.65\end{array}$ & $\begin{array}{l}0.99 \\
0.63 \\
0.74 \\
0.85 \\
0.55 \\
0.51 \\
0.87\end{array}$ & $\begin{array}{c}113.07 \\
113200 \\
-104.42 \\
399.33 \\
157.64 \\
160.45 \\
5.7\end{array}$ & 행 & $\begin{array}{l}0.05 \\
0.31 \\
0.26 \\
.0 .39 \\
0.39 \\
0.35 \\
0.21\end{array}$ & $\frac{n}{2}$ & $\begin{array}{l}78.81 \\
7.4 .42 \\
-72.60 \\
275.94 \\
112.65 \\
114.76 \\
4.72\end{array}$ & $\begin{array}{l}0.45 \\
0.55 \\
0.50 \\
0.87 \\
0.54 \\
0.59 \\
0.76\end{array}$ & $\begin{array}{c}118.71 \\
114.63 \\
-20890 \\
413.91 \\
168.97 \\
172.15 \\
7.08\end{array}$ & 总 \\
\hline
\end{tabular}




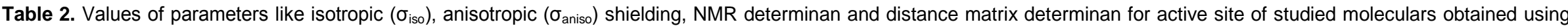
different methods.

\begin{tabular}{|c|c|c|c|c|c|c|c|c|c|c|c|c|c|c|c|c|c|c|c|c|c|c|c|c|c|}
\hline \multirow{2}{*}{\multicolumn{2}{|c|}{$\begin{array}{l}\text { Method } \\
\text { Basis set }\end{array}$}} & \multicolumn{12}{|l|}{ HF } & \multirow{2}{*}{\multicolumn{4}{|c|}{\begin{tabular}{|l|} 
B3LYP \\
6-31G \\
\end{tabular}}} & \multicolumn{4}{|c|}{ BLYP } & \multicolumn{4}{|c|}{ B3PW91 } \\
\hline & & \multicolumn{4}{|c|}{ 6-31G } & \multicolumn{4}{|c|}{ 6-31G* } & \multicolumn{4}{|c|}{$6-31+G$} & & & & & & & & & & & & \\
\hline 节 & 范 & 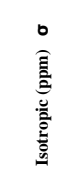 & 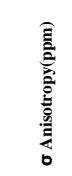 & 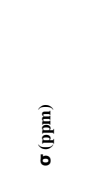 & 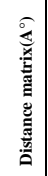 & 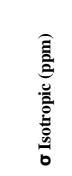 & 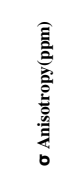 & $\begin{array}{l}\widehat{\bar{E}} \\
\text { 产 } \\
0\end{array}$ & 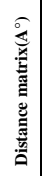 & 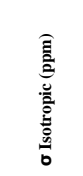 & 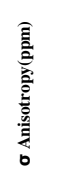 & $\begin{array}{l}\overline{\bar{E}} \\
\text { 言 } \\
0\end{array}$ & 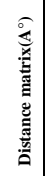 & 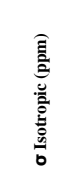 & 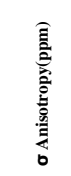 & 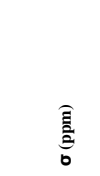 & 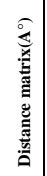 & 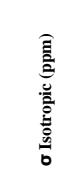 & 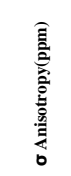 & 言 & 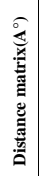 & 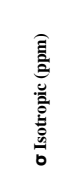 & 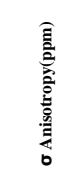 & $\begin{array}{l}\widehat{\bar{E}} \\
0 \\
0\end{array}$ & 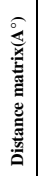 \\
\hline 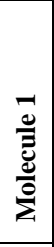 & $\begin{array}{l}\mathrm{N}(1) \\
\mathrm{C}(2) \\
\mathrm{N}(3) \\
\mathrm{C}(4) \\
\mathrm{C}(5) \\
\mathrm{C}(6) \\
\mathrm{C}(9) \\
\end{array}$ & $\begin{array}{l}127.30 \\
50.54 \\
137.20 \\
35.53 \\
98.62 \\
33.50 \\
-5.50 \\
\end{array}$ & $\begin{array}{l}70.34 \\
116.90 \\
62.23 \\
199.37 \\
116.60 \\
145.65 \\
212.40 \\
\end{array}$ & $\begin{array}{l}1.51 \mathrm{E}+06 \\
-6.34 \mathrm{E}+05 \\
1.81 \mathrm{E}+06 \\
-2.78 \mathrm{E}+05 \\
4.96 \mathrm{E}+05 \\
-6.09 \mathrm{E}+05 \\
5.62 \mathrm{E}+05 \\
\end{array}$ & 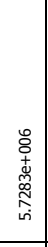 & $\begin{array}{c}133.70 \\
58.91 \\
142.97 \\
36.01 \\
99.74 \\
43.14 \\
11.30 \\
\end{array}$ & $\begin{array}{l}43.55 \\
86.94 \\
61.33 \\
190 \\
101.30 \\
115.32 \\
173.41 \\
\end{array}$ & 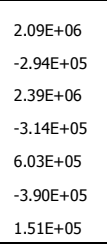 & 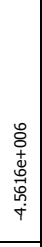 & $\begin{array}{l}126.44 \\
48.68 \\
138.07 \\
35.89 \\
98.12 \\
33.75 \\
-6.60 \\
\end{array}$ & $\begin{array}{c}66.19 \\
117.18 \\
65.16 \\
198.46 \\
115.79 \\
143.88 \\
213.93 \\
\end{array}$ & $\begin{array}{c}1.52 \mathrm{E}+06 \\
-5.88 \mathrm{E}+05 \\
1.84 \mathrm{E}+06 \\
-2.72 \mathrm{E}+06 \\
4.81 \mathrm{E}+05 \\
-5.77 \mathrm{E}+05 \\
3.09 \mathrm{E}+07 \\
\end{array}$ & 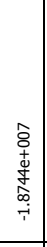 & $\begin{array}{l}95.00 \\
50.43 \\
104.56 \\
38.12 \\
84.29 \\
34.00 \\
-6.44 \\
\end{array}$ & $\begin{array}{l}67.96 \\
79.05 \\
68.47 \\
164.91 \\
114.02 \\
105.76 \\
178.58 \\
\end{array}$ & $\begin{array}{l}3.76 \mathrm{E}+05 \\
-4.13 \mathrm{E}+05 \\
5.45 \mathrm{E}+05 \\
-1.10 \mathrm{E}+05 \\
2.98 \mathrm{E}+05 \\
-4.53 \mathrm{E}+05 \\
3.70 \mathrm{E}+05 \\
\end{array}$ & 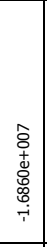 & $\begin{array}{c}82.64 \\
49.00 \\
91.93 \\
36.74 \\
79.17 \\
32.75 \\
-7.72 \\
\end{array}$ & $\begin{array}{l}65.74 \\
66.86 \\
71.33 \\
154.19 \\
110.37 \\
91.63 \\
166.25 \\
\end{array}$ & $\begin{array}{l}-1.61 E+05 \\
-3.49 E+05 \\
2.45 E+05 \\
-6.98 E+04 \\
2.40 E+05 \\
-4.02 E+05 \\
3.08 E+05 \\
\end{array}$ & 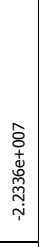 & $\begin{array}{l}99.61 \\
54.24 \\
109.08 \\
42.08 \\
88.05 \\
37.84 \\
-2.97 \\
\end{array}$ & $\begin{array}{l}71.28 \\
78.39 \\
64.93 \\
164.28 \\
113.81 \\
104.92 \\
178.27 \\
\end{array}$ & $\begin{array}{l}4.93 \mathrm{E}+05 \\
-3.91 \mathrm{E}+05 \\
6.83 \mathrm{E}+05 \\
-1.27 \mathrm{E}+05 \\
3.67 \mathrm{E}+05 \\
-4.55 \mathrm{E}+05 \\
3.33 \mathrm{E}+05 \\
\end{array}$ & 8 \\
\hline 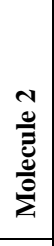 & $\begin{array}{l}\mathrm{C}(1) \\
\mathrm{C}(2) \\
\mathrm{N}(3) \\
\mathrm{C}(4) \\
\mathrm{N}(5) \\
\mathrm{C}(6) \\
\mathrm{C}(7) \\
\end{array}$ & $\begin{array}{l}96.40 \\
82.34 \\
178.94 \\
47.06 \\
205.31 \\
168.77 \\
66.20 \\
\end{array}$ & $\begin{array}{l}126.78 \\
120.34 \\
61.57 \\
131.06 \\
109.45 \\
32.79 \\
195.73 \\
\end{array}$ & $\begin{array}{c}-9.36 \mathrm{E}+03 \\
-3.26 \mathrm{E}+05 \\
4.98 \mathrm{E}+06 \\
-4.73 \mathrm{E}+05 \\
7.58 \mathrm{E}+06 \\
4.75 \mathrm{E}+06 \\
-2.4 \mathrm{E}+05 \\
\end{array}$ & 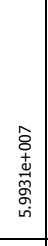 & $\begin{array}{l}96.56 \\
78.05 \\
179.37 \\
55.87 \\
206.04 \\
162.43 \\
65.03 \\
\end{array}$ & $\begin{array}{l}115.99 \\
115.23 \\
53.93 \\
100.02 \\
104.72 \\
30.75 \\
189.24 \\
\end{array}$ & $\begin{array}{l}1.36 E+05 \\
-2.85 E+05 \\
5.32 E+06 \\
-2.47 E+05 \\
8.00 E+06 \\
4.23 E+06 \\
-2.03 E+05 \\
\end{array}$ & 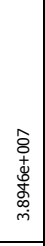 & $\begin{array}{l}95.06 \\
82.25 \\
179.20 \\
45.01 \\
203.20 \\
167.97 \\
64.45 \\
\end{array}$ & $\begin{array}{l}128.29 \\
119.31 \\
64.27 \\
133.77 \\
108.64 \\
34.25 \\
196.70 \\
\end{array}$ & $\begin{array}{l}-5.68 \mathrm{E}+04 \\
-3.15 E+05 \\
4.99 \mathrm{E}+06 \\
-4.33 \mathrm{E}+05 \\
7.30 \mathrm{E}+06 \\
4.67 \mathrm{E}+06 \\
-2.55 \mathrm{E}+05 \\
\end{array}$ & 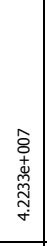 & $\begin{array}{l}89.62 \\
77.56 \\
146.25 \\
49.30 \\
177.14 \\
149.40 \\
61.72 \\
\end{array}$ & $\begin{array}{l}113.06 \\
106.01 \\
56.60 \\
93.49 \\
105.91 \\
33.77 \\
178.54 \\
\end{array}$ & $\begin{array}{l}2.04 \mathrm{E}+05 \\
-4.11 \mathrm{E}+04 \\
2.56 \mathrm{E}+06 \\
-3.72 \mathrm{E}+05 \\
4.79 \mathrm{E}+06 \\
3.28 \mathrm{E}+06 \\
-1.02 \mathrm{E}+05 \\
\end{array}$ & 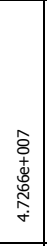 & $\begin{array}{l}- \\
- \\
- \\
- \\
-\end{array}$ & $\begin{array}{l}- \\
- \\
. \\
. \\
.\end{array}$ & $\begin{array}{l}- \\
- \\
- \\
-\end{array}$ & 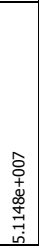 & $\begin{array}{l}93.16 \\
80.31 \\
150.30 \\
53.03 \\
182.13 \\
152.99 \\
65.09 \\
\end{array}$ & $\begin{array}{l}112.32 \\
106.17 \\
55.70 \\
92.44 \\
106.48 \\
32.85 \\
177.60\end{array}$ & $\begin{array}{l}2.79 \mathrm{E}+05 \\
986.54 \\
2.81 \mathrm{E}+06 \\
-3.51 \mathrm{E}+05 \\
5.23 \mathrm{E}+06 \\
3.52 \mathrm{E}+06 \\
-9.21 \mathrm{E}+04\end{array}$ & $\hat{o}$ \\
\hline 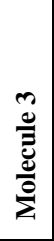 & $\begin{array}{l}\mathrm{C}(1) \\
\mathrm{C}(2) \\
\mathrm{N}(3) \\
\mathrm{C}(4) \\
\mathrm{N}(5) \\
\mathrm{C}(6) \\
\mathrm{C}(7)\end{array}$ & $\begin{array}{l}96.21 \\
63.84 \\
172.95 \\
47.71 \\
203.51 \\
172.19 \\
158.94 \\
\end{array}$ & $\begin{array}{l}131.18 \\
156.32 \\
56.96 \\
126.19 \\
107.59 \\
29.55 \\
184.09\end{array}$ & $\begin{array}{l}2.95 E+05 \\
-5.74 E+05 \\
4.52 E+06 \\
-5.53 E+05 \\
7.24 E+06 \\
5.05 E+06 \\
-4.13 E+04\end{array}$ & 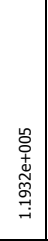 & $\begin{array}{l}97.76 \\
60.55 \\
175.12 \\
58.15 \\
205.56 \\
165.62 \\
23.73 \\
\end{array}$ & $\begin{array}{l}116.95 \\
149.00 \\
38.97 \\
94.79 \\
102.18 \\
29.84 \\
143.42 \\
\end{array}$ & $\begin{array}{l}4.3 E+05 \\
-5.28 E+05 \\
5.00 E+06 \\
-2.71 E+05 \\
7.82 E+06 \\
4.49 E+06 \\
-1.91 E+05\end{array}$ & 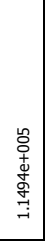 & $\begin{array}{l}94.49 \\
63.27 \\
173.18 \\
46.12 \\
201.41 \\
172.05 \\
8.32 \\
\end{array}$ & $\begin{array}{l}132.30 \\
155.79 \\
54.88 \\
127.99 \\
107.24 \\
29.89 \\
183.89 \\
\end{array}$ & $\begin{array}{l}2.75 E+05 \\
-5.46 E+05 \\
4.54 E+06 \\
-5.17 E+05 \\
6.9 E+06 \\
5.04 E+06 \\
-2.55 E+06\end{array}$ & 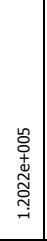 & $\begin{array}{l}84.34 \\
59.71 \\
139.43 \\
49.63 \\
173.34 \\
151.97 \\
10.72 \\
\end{array}$ & $\begin{array}{l}122.48 \\
134.57 \\
56.24 \\
88.33 \\
98.27 \\
33.95 \\
145.87\end{array}$ & $\begin{array}{l}2.44 \mathrm{E}+05 \\
-2.01 \mathrm{E}+05 \\
2.21 \mathrm{E}+06 \\
-4.16 \mathrm{E}+05 \\
4.38 \mathrm{E}+06 \\
3.45 \mathrm{E}+06 \\
-7.59 \mathrm{E}+04\end{array}$ & 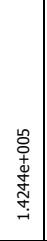 & $\begin{array}{l}79.98 \\
57.65 \\
126.93 \\
48.83 \\
161.10 \\
143.14 \\
11.29 \\
\end{array}$ & $\begin{array}{l}118.48 \\
126.15 \\
53.84 \\
75.89 \\
93.07 \\
36.84 \\
131.76\end{array}$ & $\begin{array}{l}2.05 E+05 \\
-1.26 E+05 \\
1.61 \mathrm{E}+06 \\
-3.68 \mathrm{E}+05 \\
3.49 \mathrm{E}+06 \\
2.87 \mathrm{E}+06 \\
-1.01 \mathrm{E}+05\end{array}$ & 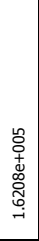 & $\begin{array}{l}88.03 \\
62.68 \\
143.88 \\
53.38 \\
178.10 \\
155.58 \\
146.99\end{array}$ & $\begin{array}{l}122.07 \\
134.87 \\
59.00 \\
87.49 \\
97.48 \\
32.75 \\
147.09\end{array}$ & $\begin{array}{l}3.08 E+05 \\
-1.89 E+05 \\
2.47 E+06 \\
-3.95 E+05 \\
4.78 E+06 \\
3.71 E+06 \\
-7.82 E+04\end{array}$ & 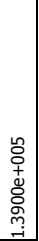 \\
\hline 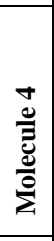 & $\begin{array}{l}C(1) \\
C(2) \\
N(3) \\
H(10) \\
C(4) \\
N(5) \\
H(11) \\
\end{array}$ & $\begin{array}{l}62.79 \\
72.08 \\
142.02 \\
26.58 \\
60.69 \\
-19.28 \\
25.28 \\
\end{array}$ & $\begin{array}{l}155.79 \\
160.64 \\
81.65 \\
11.74 \\
122.93 \\
415.85 \\
4.27 \\
\end{array}$ & $\begin{array}{c}-3.98 \mathrm{E}+04 \\
-1.80 \mathrm{E}+05 \\
2.11 \mathrm{E}+06 \\
1.73 \mathrm{E}+04 \\
-4.03 \mathrm{E}+05 \\
3.94 \mathrm{E}+06 \\
1.60 \mathrm{E}+04 \\
\end{array}$ & 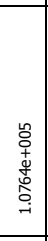 & $\begin{array}{l}63.18 \\
72.50 \\
147.60 \\
25.72 \\
63.55 \\
20.02 \\
24.98 \\
\end{array}$ & $\begin{array}{l}151.93 \\
154.02 \\
73.89 \\
11.03 \\
111.55 \\
374.36 \\
5.71 \\
\end{array}$ & $\begin{array}{l}-3.50 \mathrm{E}+04 \\
-1.27 \mathrm{E}+05 \\
2.54 \mathrm{E}+06 \\
1.54 \mathrm{E}+04 \\
-2.46 \mathrm{E}+05 \\
1.63 \mathrm{E}+06 \\
1.51 \mathrm{E}+04 \\
\end{array}$ & 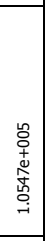 & $\begin{array}{l}61.39 \\
71.45 \\
143.86 \\
26.25 \\
59.25 \\
-19.43 \\
25.18 \\
\end{array}$ & $\begin{array}{l}157.98 \\
161.50 \\
79.86 \\
10.58 \\
124.96 \\
410.78 \\
4.28 \\
\end{array}$ & $\begin{array}{l}-4.71 \mathrm{E}+04 \\
-1.80 \mathrm{E}+05 \\
2.22 \mathrm{E}+06 \\
1.69 \mathrm{E}+04 \\
-3.99 \mathrm{E}+05 \\
3.91 \mathrm{E}+06 \\
1.58 \mathrm{E}+04 \\
\end{array}$ & 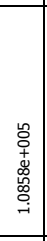 & $\begin{array}{l}53.96 \\
65.50 \\
117.10 \\
25.41 \\
59.88 \\
-20.68 \\
25.28 \\
\end{array}$ & $\begin{array}{l}136.76 \\
139.45 \\
79.13 \\
9.22 \\
94.56 \\
379.38 \\
3.96 \\
\end{array}$ & $\begin{array}{l}-1.46 \mathrm{E}+04 \\
-6.35 \mathrm{E}+04 \\
9.99 \mathrm{E}+05 \\
1.55 \mathrm{E}+04 \\
-1.53 \mathrm{E}+05 \\
3.15 \mathrm{E}+06 \\
1.60 \mathrm{E}+04 \\
\end{array}$ & 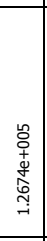 & \begin{tabular}{l|}
50.13 \\
62.07 \\
108.35 \\
25.07 \\
58.00 \\
-22.67 \\
25.23 \\
\end{tabular} & $\begin{array}{l}130.27 \\
132.85 \\
75.13 \\
8.46 \\
85.74 \\
363.26 \\
3.66 \\
\end{array}$ & $\begin{array}{l}-1.28 \mathrm{E}+04 \\
-4.91 \mathrm{E}+04 \\
7.27 \mathrm{E}+05 \\
1.50 \mathrm{E}+04 \\
-1.14 \mathrm{E}+05 \\
2.90 \mathrm{E}+06 \\
1.59 \mathrm{E}+04 \\
\end{array}$ & 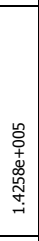 & $\begin{array}{l}57.67 \\
69.25 \\
120.56 \\
25.30 \\
62.86 \\
-14.61 \\
25.17 \\
\end{array}$ & $\begin{array}{l}135.18 \\
137.53 \\
81.27 \\
9.67 \\
94.10 \\
378.03 \\
4.30 \\
\end{array}$ & $\begin{array}{c}-1.10 E+03 \\
-3.22 E+04 \\
1.14 E+06 \\
1.52 E+04 \\
-1.23 E+05 \\
2.77 E+05 \\
1.57 E+04 \\
\end{array}$ & 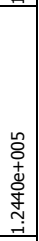 \\
\hline 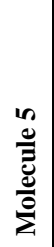 & $\begin{array}{l}\mathrm{C}(1) \\
\mathrm{C}(2) \\
\mathrm{C}(4) \\
\mathrm{N}(5) \\
\mathrm{C}(6) \\
\mathrm{C}(9) \\
\mathrm{H}(17)\end{array}$ & $\begin{array}{l}59.53 \\
70.31 \\
61.56 \\
-39.02 \\
7.18 \\
4.81 \\
30.65\end{array}$ & $\begin{array}{l}137.28 \\
127.26 \\
125.95 \\
447.94 \\
198.18 \\
204.33 \\
8.65\end{array}$ & $\begin{array}{l}-4.08 E+04 \\
-4.91 E+04 \\
-2.60 \mathrm{E}+05 \\
5.29 \mathrm{E}+06 \\
1.07 \mathrm{E}+05 \\
2.57 \mathrm{E}+05 \\
2.80 \mathrm{E}+04\end{array}$ & 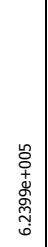 & $\begin{array}{l}58.69 \\
70.51 \\
65.12 \\
2.26 \\
21.44 \\
19.71 \\
30.33\end{array}$ & $\begin{array}{l}131.13 \\
121.51 \\
114.30 \\
404.81 \\
159.32 \\
165.13 \\
7.71\end{array}$ & $\begin{array}{l}-5.50 E+04 \\
-3.52 E+04 \\
-1.41 E+05 \\
2.56 E+06 \\
-7.75 E+04 \\
1.17 E+08 \\
2.72 E+04\end{array}$ & 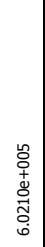 & $\begin{array}{l}59.28 \\
70.99 \\
59.91 \\
-37.89 \\
7.42 \\
5.11 \\
30.54\end{array}$ & $\begin{array}{l}139.54 \\
128.63 \\
128.31 \\
445.13 \\
199.91 \\
205.85 \\
8.49\end{array}$ & $\begin{array}{c}-5.18 \mathrm{E}+04 \\
-3.72 \mathrm{E}+04 \\
-2.69 \mathrm{E}+05 \\
5.19 \mathrm{E}+06 \\
1.36 \mathrm{E}+05 \\
2.83 \mathrm{E}+05 \\
2.77 \mathrm{E}+04\end{array}$ & 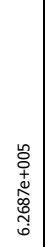 & $\begin{array}{l}52.38 \\
61.90 \\
58.67 \\
-44.79 \\
2.21 \\
2.91 \\
29.95\end{array}$ & $\begin{array}{l}119.78 \\
116.61 \\
99.10 \\
415.93 \\
168.57 \\
172.25 \\
6.56\end{array}$ & $\begin{array}{l}-2.57 E+03 \\
-1.06 \mathrm{E}+04 \\
-9.56 \mathrm{E}+04 \\
4.44 \mathrm{E}+06 \\
1.73 \mathrm{E}+05 \\
2.05 \mathrm{E}+05 \\
2.64 \mathrm{E}+04\end{array}$ & 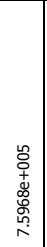 & \begin{tabular}{l|}
49.79 \\
58.69 \\
55.91 \\
-48.59 \\
-0.25 \\
1.50 \\
29.68
\end{tabular} & $\begin{array}{l}113.07 \\
113.40 \\
91.12 \\
399.33 \\
157.64 \\
160.45 \\
5.77\end{array}$ & $\begin{array}{l}-1.21 \mathrm{E}+04 \\
-1.85 \mathrm{E}+04 \\
-7.60 \mathrm{E}+04 \\
4.10 \mathrm{E}+06 \\
1.65 \mathrm{E}+05 \\
1.73 \mathrm{E}+05 \\
2.57 \mathrm{E}+04\end{array}$ & $\begin{array}{l}\text { 峉 } \\
+ \\
\mathbf{0} \\
\mathbf{0} \\
\infty \\
\infty\end{array}$ & $\begin{array}{l}55.94 \\
65.38 \\
61.71 \\
-38.82 \\
5.10 \\
5.94 \\
29.82\end{array}$ & $\begin{array}{l}118.21 \\
114.63 \\
98.27 \\
413.91 \\
168.97 \\
172.15 \\
7.08\end{array}$ & $\begin{array}{l}1.24 \mathrm{E}+04 \\
1.67 \mathrm{E}+04 \\
-6.90 \mathrm{E}+04 \\
3.94 \mathrm{E}+06 \\
1.52 \mathrm{E}+05 \\
1.78 \mathrm{E}+05 \\
2.59 \mathrm{E}+04\end{array}$ & 영 \\
\hline
\end{tabular}


the methods, except HF/6-31G*, for the $C_{2}$ and $C_{7}$ is the greatest value. In HF/6-31 $\mathrm{G}^{*}, \mathrm{~N}_{5}$ and $\mathrm{C}_{7}$ have the greatest $\eta$ value. For Molecule $4, \eta$ at all of the methods has the positive value. Also at all of the methods, $\mathrm{H}_{10}$ and $\mathrm{H}_{11}$ atoms are the greatest $\eta$ values. Finally in Molecule $5, \eta$ at all of the methods has the positive value. In HF/6-31G ,HF/6-31+G and B3PW91/6-31G levels, the $\eta$ value for the $\mathrm{C}_{4}$ and $\mathrm{N}_{5}$ is the greatest value and for the $\mathrm{C}_{1}$ is the least value, while at $H F / 6-31 G^{*}$, B3LYP/6-31G and BLYP/6-31G the $\eta$ value for the $N_{5}$ and $H_{17}$ is the greatest value and for the $C_{1}$ is the least value.

The results from Table 1 indicate that in Molecular 1 at $\mathrm{HF} / 6-31 \mathrm{G}$ level, the chemical shift anisotropy $(\Delta \sigma)$ value for the $\mathrm{N}_{1}, \mathrm{C}_{2}$ and $\mathrm{N}_{3}$ atoms is negative and for the $\mathrm{C}_{4}, \mathrm{C}_{5}$, $\mathrm{C}_{6}$ and $\mathrm{C}_{9}$ atoms is positive. In the other methods the $\Delta \sigma$ value for the $N_{1}, C_{2}, N_{3}$ and $C_{6}$ atoms is negative and for the $\mathrm{C}_{4}, \mathrm{C}_{5}$ and $\mathrm{C}_{9}$ atoms is positive. In Molecule 2 at $\mathrm{HF} / 6-31 \mathrm{G}$ and HF/6-31+G levels, $\Delta \sigma$ value for the $\mathrm{C}_{1}, \mathrm{C}_{2}$ $, \mathrm{N}_{3}, \mathrm{C}_{4}$ and $\mathrm{N}_{5}$ atoms is negative and for the $\mathrm{C}_{6}$ and $\mathrm{C}_{7}$ atoms is positive. While at $\mathrm{HF} / 6-31 \mathrm{G}^{*}, \mathrm{~B} 3 \mathrm{LYP}$ and B3PW91 $\Delta \sigma$ value for the $C_{1}, C_{2}, N_{3}$ and $C_{4}$ atoms is negative and for the $\mathrm{N}_{5}, \mathrm{C}_{6}$ and $\mathrm{C}_{7}$ atoms is positive. In Molecule 3 in all methods $\Delta \sigma$ value for the $N_{3}$ and $N_{5}$ atoms is negative and for the $C_{1}$ and $C_{7}$ atoms is positive. In Molecule 4 at HF/6-31G*, HF/6-31+G, B3LYP, BLYP and B3PW91 methods $\Delta \sigma$ value for the $N_{3}, C_{4}$ and $H_{11}$ atoms is negative, while in $\mathrm{HF} / 6-31 \mathrm{G}$, for the $\mathrm{N}_{3}$ and $\mathrm{C}_{4}$ atoms is negative. In Molecule 5 at all methods $\Delta \sigma$ value for the $\mathrm{N}_{5}$ atom is negative and other atoms are positive.

Dipole orientation (dipole moment) that reported in Table 1 , in all molecules $(1,2,3,4,5)$ for $H F / 6-31+G$ is the greatest value. At all molecules for BLYP/6-31G, the dipole moment value is least, except Molecule 5 that for $\mathrm{HF} / 6-31 \mathrm{G}^{*}$, the dipole moment value is least.

$\Delta \mathrm{E}(\mathrm{kcal} / \mathrm{mol})$ that reported in Table 1 , for all molecules at $\mathrm{HF} / 6-31 \mathrm{G}$ is zero. Also in all molecules for B3LYP method, $\Delta \mathrm{E}$ has most negative value except Molecule 1 that in $\mathrm{HF} / 6-31 \mathrm{G}$ has most negative value.

As shown in Table 2, in Molecule 1, in all methods except HF/6-31G, $\sigma_{\text {iso }}\left(\sigma\right.$ isotropic (ppm) ) for $C_{9}$ is negative, but for other atoms is positive. In HF/6-31G level, $\sigma_{\text {iso }}$ for all atoms is positive. In Molecule 2, at all methods, $\sigma_{\text {iso }}$ for all atoms is positive, and in BLYP method has not response. In Molecule 3, at all methods, $\sigma_{\text {iso }}$ for all atoms is positive. In Molecules 4 and 5 , at all methods, except HF/6-31G*, $\sigma_{\text {iso }}$ for $N_{5}$ is negative, but for other atoms is positive. In HF/6-31G* level, $\sigma_{\text {iso }}$ for all atoms is positive.

In all molecule $(1,2,3,4,5), \sigma_{\text {aniso }}(\sigma$ anisotropic (ppm), at all methods, for all atoms has positive value. In Molecule 1 in all methods, $C_{9}$ has greatest value, in Molecules 2 and 3 in all methods, $C_{7}$ has greatest value and in Molecules 4 and 5 in all methods, $N_{5}$ has greatest value.

As pointed in Table 2, distance matrix value in Molecule 1, at B3PW91 method is biggest negative amount. In Molecule 2, in all methods, distance matrix has positive amounts and in HF/6-31G has biggest amount. In Molecules 3, 4 and 5 in all methods, distance matrix has has positive amounts and in B has BLYP methods biggest amount.

Table $3 a$ and $b$ show share of orbitals contribute in the bonds (BD for 2-center bond and $\mathrm{BD}^{*}$ for 2-center anti bonding. According to Table $3 \mathrm{a}$, in Molecule 1 for HF/6$31 \mathrm{G}$, in the $\mathrm{N}_{1}-\mathrm{C}_{2}$ bond, $\mathrm{BD}=0.7858 \mathrm{sp}^{2.08}+0.6184 \mathrm{sp}^{2.02}$ reported. Polarization coefficients of the $\mathrm{N}_{1}-\mathrm{C}_{2}$ bond $\mathrm{N}_{1}=$ 0.7858 and $\mathrm{C}_{2}=0.6184$ reported, that sizes of these coefficients show the importance of the hybrid of $N_{1}$ in the formation of the bond, while in the $\mathrm{N}_{1}-\mathrm{C}_{6}$ bond $\mathrm{BD}=$ $0.7948 \mathrm{sp}^{1.98}+0.6069 \mathrm{sp}^{2.20}$ reported. Polarization coefficients of the $\mathrm{N}_{1}-\mathrm{C}_{6}$ bond $\mathrm{N}_{1}=0.7948$ and $\mathrm{C}_{2}=0.6069$ reported, that sizes of these coefficients show the importance of the hybrid of $N_{1}$ in the formation of the bond. In Molecule 2 for HF/6-31G, in the $\mathrm{C}_{2}-\mathrm{N}_{3}$ bond, polarization coefficients of this bond $\mathrm{C}_{2}=0.6198$ and $\mathrm{N}_{3}=0.7848$ reported, that sizes of these coefficients show the importance of the hybrid of $\mathrm{N}_{3}$ in the formation of the bond, while in the $\mathrm{N}_{5}-\mathrm{C}_{6}$ bond $\mathrm{N}_{5}=0.7835$ and $\mathrm{C}_{6}$ $=0.6214$ reported, that sizes of these coefficients show the importance of the hybrid of $\mathrm{N}_{5}$ in the formation of this bond. In Molecule 3 for HF/6-31G, in the $\mathrm{C}_{4}-\mathrm{N}_{5}$ bond, polarization coefficients of this bond $\mathrm{C}_{4}=0.6208$ and $\mathrm{N}_{5}=$ 0.7840 reported, that sizes of these coefficients show the importance of the hybrid of $\mathrm{N}_{5}$ in the formation of the bond, while in the $\mathrm{C}_{4}-\mathrm{N}_{3}$ bond $\mathrm{C}_{4}=0.6208$ and $\mathrm{N}_{3}=$ 0.7840 reported, that sizes of these coefficients show the importance of the hybrid of $\mathrm{N}_{3}$ in the formation of this bond. In Molecule 4 for HF/6-31G, in the $\mathrm{C}_{2}-\mathrm{N}_{3}$ bond, polarization coefficients of this bond $\mathrm{C}_{2}=0.6322$ and $\mathrm{N}_{3}=$ 0.7748 and for the $\mathrm{N}_{3}-\mathrm{C}_{4}$ bond polarization coefficients $\mathrm{N}_{3}$ $=0.7896$ and $C_{2}=0.6136$ reported, that sizes of these coefficients show the importance of the hybrid of $\mathrm{N}_{3}$ in the formation of the bonds. In Molecule 5 for HF/6-31G, at the $\mathrm{C}_{2}-\mathrm{N}_{3}$ bond, polarization coefficients of this bond $\mathrm{C}_{2}=$ 0.6372 and $\mathrm{N}_{3}=0.7707$ and for the $\mathrm{N}_{3}-\mathrm{C}_{4}$ bond polarization coefficients $\mathrm{N}_{3}=0.7922$ and $\mathrm{C}_{2}=0.6102$ reported, that sizes of these coefficients show the importance of the hybrid of $\mathrm{N}_{3}$ in the formation of these bonds reported. As shown in Table $3 a$ and $b$, for the whole molecular, in all methods at the $\mathrm{C}-\mathrm{N}$ bond, a polarization coefficient for $\mathrm{N}$ is biggest.

Chemical shifts $(\sigma)$ for each of the atom are shown in Figure 2. As shown in Figure 2, in Molecule 1 at all methods biggest signals are for atoms $\mathrm{O}_{11}$ and $\mathrm{O}_{7}$. Figure 3 shows that in Molecule 2 at all methods biggest signal is watched for the atoms $\mathrm{N}_{3}, \mathrm{~N}_{5}$ and $\mathrm{O}_{8}$, except BLYP/6$31 \mathrm{G}$ that is not response. The results from Figure 4 indicate that in Molecule 3 at all methods biggest signal is for atom $\mathrm{O}_{8}$ (aldhyd). Figure 5 shows diagrams which show chemical shifts for each of the atom at the Molecule 4 . According to Figure 5 biggest signals are for atoms $\mathrm{N}_{3}$ and $N_{5}$. At all methods $\sigma$ value for $N_{5}$ is more than $N_{3}$, but at $\mathrm{HF} / 6-31 \mathrm{G}^{*}, \sigma$ value for $\mathrm{N}_{3}$ is more than $\mathrm{N}_{5}$. As shown in Figure 6, in Molecule 5 at all methods biggest signals are for atoms $\mathrm{O}_{10}$ and $\mathrm{O}_{11}$. Figures 7 to 11 compare graphs of chemical shifts for molecule with different methods. 
Table 3a. Relative natural bond orbital( NBO) for several active bond in studied molecules by : HF method with 6-31G,6-31G* and 6-31+G basis set.

\begin{tabular}{|c|c|c|c|c|c|c|c|}
\hline \multirow{2}{*}{\multicolumn{2}{|c|}{$\begin{array}{c}\text { Method } \\
\text { Basis set }\end{array}$}} & \multicolumn{6}{|c|}{ HF } \\
\hline & & \multicolumn{2}{|c|}{$6-31 G$} & \multicolumn{2}{|c|}{$6-31+G$} & \multicolumn{2}{|c|}{$6-31 G^{*}$} \\
\hline Name & Atoms & Bonding & Antibonding & Bonding & Antibonding & Bonding & Antibonding \\
\hline \multirow{5}{*}{$\begin{array}{l}\bar{\Phi} \\
\frac{0}{J} \\
\frac{\delta}{0} \\
\bar{\Sigma}\end{array}$} & $\mathrm{N} 1-\mathrm{C} 2$ & $0.7858 \mathrm{sp}^{2.08}+0.6184 \mathrm{sp}^{2.02}$ & $0.6184 \mathrm{sp}^{2.08}-0.7858 \mathrm{sp}^{2.02}$ & $0.7840 \mathrm{sp}^{2.16}+0.6207 \mathrm{sp}^{2.03}$ & $0.6207 \mathrm{sp}^{2.16}-0.7840 \mathrm{sp}^{2.03}$ & $0.7892 \mathrm{sp}^{2.08} \mathrm{~d}^{0.01}+0.6141 \mathrm{sp}^{2.03} \mathrm{~d}^{0.01}$ & $0.6141 \mathrm{sp}^{2.08} \mathrm{~d}^{0.01}-0.7892 \mathrm{sp}^{2.02} \mathrm{~d}^{0.01}$ \\
\hline & $\mathrm{N} 1-\mathrm{C} 6$ & $0.7948 \mathrm{sp}^{1.98}+0.6069 \mathrm{sp}^{2.20}$ & $0.6069 \mathrm{sp}^{1.98}-0.7948 \mathrm{sp}^{2.20}$ & $0.7941 \mathrm{sp}^{2.03}+0.6078 \mathrm{sp}^{2.21}$ & $0.6078 \mathrm{sp}^{2.03}-0.7941 \mathrm{sp}^{2.21}$ & $0.7976 \mathrm{sp}^{1.98}+0.6031 \mathrm{sp}^{2.18} \mathrm{~d}^{0.01}$ & $0.6031 \mathrm{sp}^{1.98}-0.7976 \mathrm{sp}^{2.18} \mathrm{~d}^{0.01}$ \\
\hline & $\mathrm{N} 1-\mathrm{H} 13$ & $0.8646 \mathrm{sp}^{2.19}+0.5024 \mathrm{~s}$ & $0.5024 \mathrm{sp}^{2.19}-0.8646 \mathrm{~s}$ & $0.8662 \mathrm{sp}^{2.07}+0.4998 \mathrm{~s}$ & $0.4998 \mathrm{sp}^{2.07}-0.8662 \mathrm{~s}$ & $0.8606 \mathrm{sp}^{2.21}+0.5092 \mathrm{~s}$ & $0.5092 \mathrm{sp}^{2.21}-0.8606 \mathrm{~s}$ \\
\hline & $\mathrm{C} 4-\mathrm{C} 12$ & $0.7236 \mathrm{sp}^{2.02}+0.6902 \mathrm{sp}^{2.82}$ & $0.6902 \mathrm{sp}^{2.02}-0.7236 \mathrm{sp}^{2.82}$ & $0.7261 \mathrm{sp}^{1.98}+0.6876 \mathrm{sp}^{2.95}$ & $0.6876 \mathrm{sp}^{1.98}-0.7261 \mathrm{sp}^{2.95}$ & $0.7220 \mathrm{sp}^{2.03}+0.6919 \mathrm{sp}^{2.77} \mathrm{~d}^{0.01}$ & $0.6919 \mathrm{sp}^{2.03}-0.7220 \mathrm{sp}^{2.77} \mathrm{~d}^{0.01}$ \\
\hline & $\mathrm{C} 2-\mathrm{O} 8$ & $0.5741 \mathrm{sp}^{2.54}+0.8188 \mathrm{sp}^{1.62}$ & $0.8188 \mathrm{sp}^{2.54}-0.5741 \mathrm{sp}^{1.62}$ & $0.5940 \mathrm{sp}^{1.94}+0.8045 \mathrm{sp}^{1.52}$ & $0.8045 \mathrm{sp}^{1.94}-0.5940 \mathrm{sp}^{1.52}$ & $0.5659 \mathrm{sp}^{2.59} \mathrm{~d}^{0.01}+0.8244 \mathrm{sp}^{1.53} \mathrm{~d}^{0.01}$ & $0.8244 \mathrm{sp}^{2.59} \mathrm{~d}^{0.01}-0.5659 \mathrm{sp}^{1.53} \mathrm{~d}^{0.01}$ \\
\hline \multirow{5}{*}{ 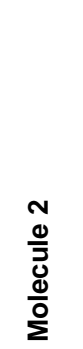 } & $\mathrm{C} 2-\mathrm{N} 3$ & $0.6198 \mathrm{sp}^{2.60}+0.7848 \mathrm{sp}^{1.88}$ & $0.7848 \mathrm{sp}^{2.60}-0.6198 \mathrm{sp}^{1.88}$ & & & $0.6194 \mathrm{sp}^{2.58} \mathrm{~d}^{0.01}+0.7851 \mathrm{sp}^{1.88}$ & $0.7851 \mathrm{sp}^{2.58} \mathrm{~d}^{0.01}-0.6194 \mathrm{sp}^{1.88}$ \\
\hline & $\mathrm{N} 3-\mathrm{C} 4$ & $0.7889 \mathrm{sp}^{1.98}+0.6145 \mathrm{sp}^{2.06}$ & $0.6145 \mathrm{sp}^{1.98}-0.7889 \mathrm{sp}^{2.06}$ & - & - & $0.7930 \mathrm{sp}^{1.96}+0.6092 \mathrm{sp}^{2.06} \mathrm{~d}^{0.01}$ & $0.6092 \mathrm{sp}^{1.96}-0.7930 \mathrm{sp}^{2.06} \mathrm{~d}^{0.01}$ \\
\hline & $\mathrm{C} 4-\mathrm{O} 8$ & $0.5690 \mathrm{sp}^{4.14}+0.8224 \mathrm{sp}^{6.08}$ & $0.8224 \mathrm{sp}^{4.14}-0.5690 \mathrm{sp}^{6.08}$ & & & $0.5716 \mathrm{sp}^{3.06} \mathrm{~d}^{0.01}+0.8205 \mathrm{sp}^{3.61} \mathrm{~d}^{0.02}$ & $0.8205 \mathrm{sp}^{3.06} \mathrm{~d}^{0.01}-0.5716 \mathrm{sp}^{3.61} \mathrm{~d}^{0.02}$ \\
\hline & N5 - C6 & $0.7835 \mathrm{sp}^{1.78}+0.6214 \mathrm{sp}^{3.18}$ & $0.6214 \mathrm{sp}^{1.78}-0.7835 \mathrm{sp}^{3.18}$ & & & $0.7844 \mathrm{sp}^{1.78}+0.6202 \mathrm{sp}^{3.13} \mathrm{~d}^{0.01}$ & $0.6202 \mathrm{sp}^{1.78}-0.7844 \mathrm{sp}^{3.13} \mathrm{~d}^{0.01}$ \\
\hline & $\mathrm{C} 1-\mathrm{C} 7$ & $0.7085 \mathrm{sp}^{2.52}+0.7057 \mathrm{sp}^{2.66}$ & $0.7057 \mathrm{sp}^{2.52}-0.7085 \mathrm{sp}^{2.66}$ & & & $0.7079 \mathrm{sp}^{2.54}+0.7063 \mathrm{sp}^{2.67}$ & $0.7063 \mathrm{sp}^{2.54}-0.7079 \mathrm{sp}^{2.67}$ \\
\hline \multirow{5}{*}{ 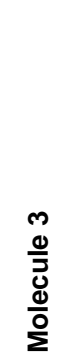 } & N3 - C4 & $0.7904 \mathrm{sp}^{1.96}+0.6126 \mathrm{sp}^{2.08}$ & $0.6126 \mathrm{sp}^{1.96}-0.7904 \mathrm{sp}^{2.08}$ & $0.7885 \mathrm{sp}^{2.04}+0.6150 \mathrm{sp}^{2.08}$ & $0.6150 \mathrm{sp}^{2.04}-0.7885 \mathrm{sp}^{2.08}$ & $0.7944 \mathrm{sp}^{1.94}+0.6074 \mathrm{sp}^{2.08} \mathrm{~d}^{0.01}$ & $0.6074 \mathrm{sp}^{1.94}-0.7944 \mathrm{sp}^{2.08} \mathrm{~d}^{0.01}$ \\
\hline & C4-N5 & $0.6208 \mathrm{sp}^{2}+0.7840 \mathrm{sp}^{2.05}$ & $0.7840 \mathrm{sp}^{2}-0.6208 \mathrm{sp}^{2.05}$ & $0.6228 \mathrm{sp}^{2.01}+0.7824 \mathrm{sp}^{2.13}$ & $0.7824 \mathrm{sp}^{2.01}-0.6228 \mathrm{sp}^{2.13}$ & $0.6153 \mathrm{sp}^{2} \mathrm{~d}^{0.01}+0.7883 \mathrm{sp}^{2.03}$ & $0.7883 \mathrm{sp}^{2} \mathrm{~d}^{0.01}-0.6153 \mathrm{sp}^{2.03}$ \\
\hline & N5 - C6 & $0.7851 \mathrm{sp}^{1.78}+0.6194 \mathrm{sp}^{3.20}$ & $0.6194 \mathrm{sp}^{1.78}-0.7851 \mathrm{sp}^{3.20}$ & $0.7869 \mathrm{sp}^{1.79}+0.6170 \mathrm{sp}^{3.29}$ & $0.6170 \mathrm{sp}^{1.79}-0.7869 \mathrm{sp}^{3.29}$ & $0.7861 \mathrm{sp}^{1.78}+0.6181 \mathrm{sp}^{3.15} \mathrm{~d}^{0.01}$ & $0.6181 \mathrm{sp}^{1.78}-0.7861 \mathrm{sp}^{3.15} \mathrm{~d}^{0.01}$ \\
\hline & $\mathrm{C} 2-\mathrm{N} 3$ & $0.6222 \mathrm{sp}^{2.56}+0.7829 \mathrm{sp}^{1.91}$ & $0.7829 \mathrm{sp}^{2.56}-0.6222 \mathrm{sp}^{1.91}$ & $0.6201 \mathrm{sp}^{2.60}+0.7845 \mathrm{sp}^{1.91}$ & $0.62017845 \mathrm{sp}^{2.60}-0.6201 \mathrm{sp}^{1.91}$ & $0.6213 \mathrm{sp}^{2.55} \mathrm{~d}^{0.01}+0.7835 \mathrm{sp}^{1.91}$ & $0.7835 \mathrm{sp}^{2.55} \mathrm{~d}^{0.01}-0.6213 \mathrm{sp}^{1.91}$ \\
\hline & $\mathrm{C} 1-\mathrm{C} 6$ & $0.7136 \mathrm{sp}^{1.99}+0.7005 \mathrm{sp}^{2.65}$ & $0.7005 \mathrm{sp}^{1.99}-0.7136 \mathrm{sp}^{2.65}$ & $0.7143 \mathrm{sp}^{1.99}+0.6999 \mathrm{sp}^{2.71}$ & $0.6999 \mathrm{sp}^{1.99}-0.7143 \mathrm{sp}^{2.71}$ & $0.7138 \mathrm{sp}^{1.98}+0.7004 \mathrm{sp}^{2.62} \mathrm{~d}^{0.01}$ & $0.7004 \mathrm{sp}^{1.98}-0.7138 \mathrm{sp}^{2.62} \mathrm{~d}^{0.01}$ \\
\hline \multirow{4}{*}{ 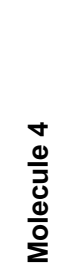 } & $\mathrm{C} 1-\mathrm{N} 5$ & $0.6468 \mathrm{sp}^{2.98}+0.7627 \mathrm{sp}^{3.23}$ & $0.7627 \mathrm{sp}^{2.98}-0.6468 \mathrm{sp}^{3.23}$ & - & - & $0.6450 \mathrm{sp}^{2.96} \mathrm{~d}^{0.01}+0.7642 \mathrm{sp}^{3.21} \mathrm{~d}^{0.01}$ & $0.7642 \mathrm{sp}^{2.96} \mathrm{~d}^{0.01}-0.6450 \mathrm{sp}^{3.21} \mathrm{~d}^{0.01}$ \\
\hline & $\mathrm{C} 2-\mathrm{N} 3$ & $0.6322 \mathrm{sp}^{3.10}+0.7748 \mathrm{sp}^{2.40}$ & $0.7748 \mathrm{sp}^{3.10}-0.6322 \mathrm{sp}^{2.40}$ & . & - & $0.6306 \mathrm{sp}^{3.08}+0.7761 \mathrm{sp}^{2.42}$ & $0.7761 \mathrm{sp}^{3.08}-0.6306 \mathrm{sp}^{2.42}$ \\
\hline & N3 $-\mathrm{C} 4$ & $0.7896 \mathrm{sp}^{2.63}+0.6136 \mathrm{sp}^{2.69}$ & $0.6136 \mathrm{sp}^{2.63}-0.7896 \mathrm{sp}^{2.69}$ & _. & - & $0.7910 \mathrm{sp}^{2.66} \mathrm{~d}^{0.01}+0.6118 \mathrm{sp}^{2.65} \mathrm{~d}^{0.01}$ & $0.6118 \mathrm{sp}^{2.66} \mathrm{~d}^{0.01}-0.7910 \mathrm{sp}^{2.65} \mathrm{~d}^{0.01}$ \\
\hline & $\mathrm{C} 1-\mathrm{C} 6$ & $0.7147 \mathrm{sp}^{1.47}+0.6994 \mathrm{sp}^{1.93}$ & $0.6994 \mathrm{sp}^{1.47}-0.7147 \mathrm{sp}^{1.93}$ & & - & $0.7140 \mathrm{sp}^{1.48}+0.7001 \mathrm{sp}^{1.92}$ & $0.7001 \mathrm{sp}^{1.48}-0.7140 \mathrm{sp}^{1.92}$ \\
\hline
\end{tabular}


Table 3a. Contd.

\begin{tabular}{|c|c|c|c|c|c|c|c|}
\hline & $\mathrm{C} 2-\mathrm{Cg}$ & $0.7160 \mathrm{sp}^{1.47}+0.6981 \mathrm{sp}^{1.95}$ & $0.6981 \mathrm{sp}^{1.47}-0.7160 \mathrm{sp}^{1.95}$ & - & - & $0.7151 \mathrm{sp}^{1.47}+0.6990 \mathrm{sp}^{1.94}$ & $0.6990 \mathrm{sp}^{1.47}-0.7151 \mathrm{sp}^{1.94}$ \\
\hline \multirow{5}{*}{ 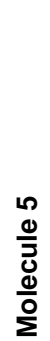 } & $\mathrm{C} 1-\mathrm{N} 5$ & $0.6529 \mathrm{sp}^{2.58}+0.7574 \mathrm{sp}^{3.27}$ & $0.7574 \mathrm{sp}^{2.58}-0.6529 \mathrm{sp}^{3.27}$ & $0.6545 \mathrm{sp}^{2.53}+0.7561 \mathrm{sp}^{3.33}$ & $0.7561 \mathrm{sp}^{2.53}-0.6545 \mathrm{sp}^{3.33}$ & $0.6507 \mathrm{sp}^{2.56} \mathrm{~d}^{0.01}+0.7593 \mathrm{sp}^{3.23} \mathrm{~d}^{0.01}$ & $0.7593 \mathrm{sp}^{2.56} \mathrm{~d}^{0.01}-0.6507 \mathrm{sp}^{3.23} \mathrm{~d}^{0.01}$ \\
\hline & $\mathrm{C} 2-\mathrm{N} 3$ & $0.6372 \mathrm{sp}^{2.74}+0.7707 \mathrm{sp}^{2.52}$ & $0.7707 \mathrm{sp}^{2.74}-0.6372 \mathrm{sp}^{2.52}$ & $0.6381 \mathrm{sp}^{2.70}+0.7700 \mathrm{sp}^{2.59}$ & $0.7700 \mathrm{sp}^{2.70}-0.6381 \mathrm{sp}^{2.59}$ & $0.6352 \mathrm{sp}^{2.71} \mathrm{~d}^{0.01}+0.7724 \mathrm{sp}^{2.52}$ & $0.7724 \mathrm{sp}^{2.71} \mathrm{~d}^{0.01}-0.6352 \mathrm{sp}^{2.52} \mathrm{~d}^{0.01}$ \\
\hline & $\mathrm{C} 4-\mathrm{N} 5$ & $0.6298 \mathrm{sp}^{1.83}+0.7767 \mathrm{sp}^{1.29}$ & $0.7767 \mathrm{sp}^{1.83}-0.6298 \mathrm{sp}^{1.29}$ & $0.6330 \mathrm{sp}^{1.82}+0.7742 \mathrm{sp}^{1.32}$ & $0.7742 \mathrm{sp}^{1.82}-0.6330 \mathrm{sp}^{1.32}$ & $0.6279 \mathrm{sp}^{1.80} \mathrm{~d}^{0.01}+0.7783 \mathrm{sp}^{1.28} \mathrm{~d}^{0.01}$ & $0.7783 \mathrm{sp}^{1.80} \mathrm{~d}^{0.01}-0.6279 \mathrm{sp}^{1.28}$ \\
\hline & $\mathrm{C} 4-\mathrm{N} 5$ & $0.6445 \mathrm{sp}+0.7646 \mathrm{sp}^{99.99}$ & $0.7646 \mathrm{sp}-0.6445 \mathrm{sp}^{99.99}$ & $0.6419 \mathrm{sp}+0.7668 \mathrm{sp}^{99.99}$ & $0.7668 \mathrm{sp}-0.6419 \mathrm{sp} \mathrm{sp}^{99.99}$ & $0.6351 \mathrm{sp}+0.7724 \mathrm{sp}^{99.99} \mathrm{~d}^{13.35}$ & $0.7724 \mathrm{sp}-0.6351 \mathrm{sp}^{99.99} \mathrm{~d}^{13.35}$ \\
\hline & N3 $-\mathrm{C} 4$ & $0.7922 \mathrm{sp}^{2.56}+0.6102 \mathrm{sp}^{2.74}$ & $0.6102 \mathrm{sp}^{2.56}-0.7922 \mathrm{sp}^{2.74}$ & $0.7905 \mathrm{sp}^{2.62}+0.6125 \mathrm{sp}^{2.77}$ & $0.6125 \mathrm{sp}^{2.62}-0.7905 \mathrm{sp}^{2.77}$ & $0.7932 \mathrm{sp}^{2.59} \mathrm{~d}^{0.01}+0.6090 \mathrm{sp}^{2.69} \mathrm{~d}^{0.01}$ & $0.6090 \mathrm{sp}^{2.59} \mathrm{~d}^{0.01}-0.7932 \mathrm{sp}^{2.69} \mathrm{~d}^{0.01}$ \\
\hline
\end{tabular}

Table 3b. Relative natural bond orbital (NBO) for several active bond in studied molecules by: B3LYP, BLYP and B3PW91 methods with 6-31G basis set.

\begin{tabular}{|c|c|c|c|c|c|c|c|}
\hline \multicolumn{2}{|c|}{ Method } & \multicolumn{2}{|c|}{ B3LYP } & \multicolumn{2}{|c|}{ BLYP } & \multicolumn{2}{|c|}{ B3PW91 } \\
\hline \multicolumn{2}{|c|}{ Basis set } & \multicolumn{6}{|c|}{ 6-31G } \\
\hline Name & Atoms & Bonding & Antibonding & Bonding & Antibonding & Bonding & Antibonding \\
\hline \multirow{6}{*}{ 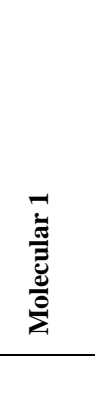 } & $\mathrm{N} 1-\mathrm{C} 2$ & $0.7842 \mathrm{sp}^{2.08}+0.6205 \mathrm{sp}^{2.00}$ & $0.6205 \mathrm{sp}^{2.08}-0.7842 \mathrm{sp}^{2.00}$ & $0.5875 \mathrm{sp}^{2.08}+0.8092 \mathrm{sp}^{1.47}$ & $0.6208 \mathrm{sp}^{2.08}-0.7839 \mathrm{sp}^{1.99}$ & $0.7839 \mathrm{sp}^{2.08}+0.6208 \mathrm{sp}^{1.99}$ & $0.6208 \mathrm{sp}^{2.08}-0.7839 \mathrm{sp}^{1.99}$ \\
\hline & $\mathrm{N} 1-\mathrm{C} 6$ & $0.7923 \mathrm{sp}^{1.99}+0.6102 \mathrm{sp}^{2.17}$ & $0.6102 \mathrm{sp}^{1.99}-0.7923 \mathrm{sp}^{2.17}$ & $0.7919 \mathrm{sp}^{2.00}+0.6106 \mathrm{sp}^{2.16}$ & $0.6106 \mathrm{sp}^{2.00}-0.7919 \mathrm{sp}^{2.16}$ & $0.7919 \mathrm{sp}^{2.00}+0.6106 \mathrm{sp}^{2.16}$ & $0.6106 \mathrm{sp}^{2.00}-0.7919 \mathrm{sp}^{2.16}$ \\
\hline & $\mathrm{N} 1-\mathrm{H} 13$ & $0.8593 \mathrm{sp}^{2.28}+0.5114 \mathrm{~s}$ & $0.5114 \mathrm{sp}^{2.28}-0.8593 \mathrm{~s}$ & $0.8566 \mathrm{sp}^{2.32}+0.5159 \mathrm{~s}$ & $0.5159 \mathrm{sp}^{2.32}-0.8566 \mathrm{~s}$ & $0.8566 \mathrm{sp}^{2.32}+0.5159 \mathrm{~s}$ & $0.5159 \mathrm{sp}^{2.32}-0.8566 \mathrm{~s}$ \\
\hline & $\mathrm{C} 4-\mathrm{C} 12$ & $0.7177 \mathrm{sp}^{2.05}+0.6964 \mathrm{sp}^{2.77}$ & $0.6964 \mathrm{sp}^{2.05}-0.7177 \mathrm{sp}^{2.77}$ & $0.7161 \mathrm{sp}^{2.05}+0.6980 \mathrm{sp}^{2.75}$ & $0.6980 \mathrm{sp}^{2.05}-0.7161 \mathrm{sp}^{2.75}$ & $0.7161 \mathrm{sp}^{2.05}+0.6980 \mathrm{sp}^{2.75}$ & $0.6980 \mathrm{sp}^{2.05}-0.7161 \mathrm{sp}^{2.75}$ \\
\hline & $\mathrm{C} 2-08$ & $0.5875 \mathrm{sp}^{2.08}+0.8092 \mathrm{sp}^{1.47}$ & $0.8092 \mathrm{sp}^{2.08}-0.5875 \mathrm{sp}^{1.47}$ & $0.5884 \mathrm{sp}^{2.06}+0.8085 \mathrm{sp}^{1.48}$ & $0.8085 \mathrm{sp}^{2.06}-0.5884 \mathrm{sp}^{1.48}$ & $0.5884 \mathrm{sp}^{2.06}+0.8085 \mathrm{sp}^{1.48}$ & $0.8085 \mathrm{sp}^{2.06}-0.5884 \mathrm{sp}^{1.48}$ \\
\hline & $\mathrm{C} 2-\mathrm{N} 3$ & $0.6228 \mathrm{sp}^{2.59}+0.7824 \mathrm{sp}^{1.89}$ & $0.7824 \mathrm{sp}^{2.59}-0.6228 \mathrm{sp}^{1.89}$ & $0.6231 \mathrm{sp}^{2.59}+0.7821 \mathrm{sp}^{1.89}$ & $0.7821 \mathrm{sp}^{2.59}-0.6231 \mathrm{sp}^{1.89}$ & $0.6232 \mathrm{sp}^{2.60}+0.7821 \mathrm{sp}^{1.90}$ & $0.7821 \mathrm{sp}^{2.60}-0.6232 \mathrm{sp}^{1.90}$ \\
\hline
\end{tabular}


Table 3b. Contd.

\begin{tabular}{|c|c|c|c|c|c|c|c|}
\hline \multirow{4}{*}{ 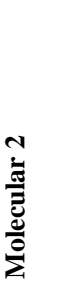 } & $\mathrm{N} 3-\mathrm{C} 4$ & $0.7869 \mathrm{sp}^{1.94}+0.6170 \mathrm{sp}^{2.04}$ & $0.6170 \mathrm{sp}^{1.94}-0.7869 \mathrm{sp}^{2.04}$ & $0.7867 \mathrm{sp}^{1.93}+0.6173 \mathrm{sp}^{2.04}$ & $0.6173 \mathrm{sp}^{1.93}-0.7867 \mathrm{sp}^{2.04}$ & $0.7870 \mathrm{sp}^{1.95}+0.6169 \mathrm{sp}^{2.05}$ & $0.6169 \mathrm{sp}^{1.95}-0.7870 \mathrm{sp}^{2.05}$ \\
\hline & $C 4-08$ & $0.5948 \mathrm{sp}^{2.18}+0.8039 \mathrm{sp}^{2.14}$ & $0.8039 \mathrm{sp}^{2.18} 0.5948 \mathrm{sp}^{2.14}$ & $0.5948 \mathrm{sp}^{2.06}+0.8039 \mathrm{sp}^{1.87}$ & $0.8039 \mathrm{sp}^{2.06}-0.5948 \mathrm{sp}^{1.87}$ & $0.5954 \mathrm{sp}^{2.20}+0.8035 \mathrm{sp}^{2.19}$ & $0.8035 \mathrm{sp}^{2.20}-0.5954 \mathrm{sp}^{2.19}$ \\
\hline & N5- C6 & $0.7783 \mathrm{sp}^{1.81}+0.6279 \mathrm{sp}^{3.15}$ & $0.6279 \mathrm{sp}^{1.81}-0.7783 \mathrm{sp}^{3.15}$ & $0.7774 \mathrm{sp}^{1.81}+0.6290 \mathrm{sp}^{3.15}$ & $0.6290 \mathrm{sp}^{1.81}-0.7774 \mathrm{sp}^{3.15}$ & $0.7779 \mathrm{sp}^{1.81}+0.6284 \mathrm{sp}^{3.16}$ & $0.6284 \mathrm{sp}^{1.81}-0.7779 \mathrm{sp}^{3.16}$ \\
\hline & $\mathrm{C} 1-\mathrm{C} 7$ & $0.7079 \mathrm{sp}^{2.54}+0.7063 \mathrm{sp}^{2.69}$ & $0.7063 \mathrm{sp}^{2.54}-0.7079 \mathrm{sp}^{2.69}$ & $0.7080 \mathrm{sp}^{2.55}+0.7062 \mathrm{sp}^{2.71}$ & $0.7062 \mathrm{sp}^{2.55}-0.7080 \mathrm{sp}^{2.71}$ & $0.7079 \mathrm{sp}^{2.54}+0.7063 \mathrm{sp}^{2.69}$ & $0.7063 \mathrm{sp}^{2.54}-0.7079 \mathrm{sp}^{2.69}$ \\
\hline \multirow{5}{*}{ 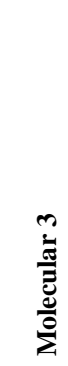 } & $\mathrm{N} 3-\mathrm{C} 4$ & $0.7880 \mathrm{sp}^{1.93}+0.6157 \mathrm{sp}^{2.06}$ & $0.6157 \mathrm{sp}^{1.93}-0.7880 \mathrm{sp}^{2.06}$ & $0.7877 \mathrm{sp}^{1.92}+0.6161 \mathrm{sp}^{2.06}$ & $0.6161 \mathrm{sp}^{1.92}-0.7877 \mathrm{sp}^{2.06}$ & $0.7881 \mathrm{sp}^{1.94}+0.6156 \mathrm{sp}^{2.06}$ & $0.6156 \mathrm{sp}^{1.94}-0.7881 \mathrm{sp}^{2.06}$ \\
\hline & $\mathrm{C} 4-\mathrm{N} 5$ & $0.6225 \mathrm{sp}^{1.98}+0.7827 \mathrm{sp}^{2.01}$ & $0.7827 \mathrm{sp}^{1.98}-0.6225 \mathrm{sp}^{2.01}$ & $0.6226 \mathrm{sp}^{1.97}+0.7825 \mathrm{sp}^{1.99}$ & $0.7825 \mathrm{sp}^{1.97}-0.6226 \mathrm{sp}^{1.99}$ & $0.6222 \mathrm{sp}^{1.98}+0.7829 \mathrm{sp}^{2.01}$ & $0.7829 \mathrm{sp}^{1.98}-0.6222 \mathrm{sp}^{2.01}$ \\
\hline & N5- C6 & $0.7800 \mathrm{sp}^{1.80}+0.6258 \mathrm{sp}^{3.18}$ & $0.6258 \mathrm{sp}^{1.80}-0.7800 \mathrm{sp}^{3.18}$ & $0.7792 \mathrm{sp}^{1.80}+0.6268 \mathrm{sp}^{3.17}$ & $0.6268 \mathrm{sp}^{1.80}-0.7792 \mathrm{sp}^{3.17}$ & $0.7797 \mathrm{sp}^{1.81}+0.6261 \mathrm{sp}^{3.19}$ & $0.6261 \mathrm{sp}^{1.81}-0.7797 \mathrm{sp}^{3.19}$ \\
\hline & $\mathrm{C} 2-\mathrm{N} 3$ & $0.6236 \mathrm{sp}^{2.57}+0.7817 \mathrm{sp}^{1.91}$ & $0.7817 \mathrm{sp}^{2.57}-0.6236 \mathrm{sp}^{1.91}$ & $0.6235 \mathrm{sp}^{2.58}+0.7819 \mathrm{sp}^{1.91}$ & $0.7819 \mathrm{sp}^{2.58}-0.6235 \mathrm{sp}^{1.91}$ & $0.6239 \mathrm{sp}^{2.58}+0.7815 \mathrm{sp}^{1.92}$ & $0.7815 \mathrm{sp}^{2.58}-0.6239 \mathrm{sp}^{1.92}$ \\
\hline & $\mathrm{C} 1-\mathrm{C} 6$ & $0.7076 \mathrm{sp}^{2}+0.7066 \mathrm{sp}^{2.62}$ & $0.7066 \mathrm{sp}^{2}-0.7076 \mathrm{sp}^{2.62}$ & $0.7060 \mathrm{sp}^{2.01}+0.7082 \mathrm{sp}^{2.60}$ & $0.7082 \mathrm{sp}^{2.01}-0.7060 \mathrm{sp}^{2.60}$ & $0.7078 \mathrm{sp}^{2}+0.7065 \mathrm{sp}^{2.62}$ & $0.7065 \mathrm{sp}^{2}-0.7078 \mathrm{sp}^{2.62}$ \\
\hline \multirow{5}{*}{ 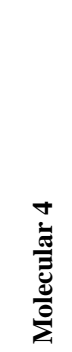 } & C1-N5 & $0.6507 \mathrm{sp}^{3.01}+0.7593 \mathrm{sp}^{3.49}$ & $0.7593 \mathrm{sp}^{3.01}-0.6507 \mathrm{sp}^{3.49}$ & $0.6515 \mathrm{sp}^{3.03}+0.7587 \mathrm{sp}^{3.57}$ & $0.7587 \mathrm{sp}^{3.03}-0.6515 \mathrm{sp}^{3.57}$ & $0.6512 \mathrm{sp}^{3.01}+0.7589 \mathrm{sp}^{3.50}$ & $0.7589 \mathrm{sp}^{3.01}-0.6512 \mathrm{sp}^{3.50}$ \\
\hline & $\mathrm{C} 2-\mathrm{N} 3$ & $0.6330 \mathrm{sp}^{3.17}+0.7742 \mathrm{sp}^{2.46}$ & $0.7742 \mathrm{sp}^{3.17}-0.6330 \mathrm{sp}^{2.46}$ & $0.6333 \mathrm{sp}^{3.19}+0.7739 \mathrm{sp}^{2.49}$ & $0.7739 \mathrm{sp}^{3.19}-0.6333 \mathrm{sp}^{2.49}$ & $0.6338 \mathrm{sp}^{3.17}+0.7735 \mathrm{sp}^{2.48}$ & $0.7735 \mathrm{sp}^{3.17}-0.6338 \mathrm{sp}^{2.48}$ \\
\hline & $\mathrm{N} 3-\mathrm{C} 4$ & $0.7888 \mathrm{sp}^{2.68}+0.6147 \mathrm{sp}^{2.73}$ & $0.6147 \mathrm{sp}^{2.68}-0.7888 \mathrm{sp}^{2.73}$ & $0.7886 \mathrm{sp}^{2.70}+0.6149 \mathrm{sp}^{2.73}$ & $0.6149 \mathrm{sp}^{2.70}-0.7886 \mathrm{sp}^{2.73}$ & $0.7888 \mathrm{sp}^{2.69}+0.6146 \mathrm{sp}^{2.74}$ & $0.6146 \mathrm{sp}^{2.69}-0.7888 \mathrm{sp}^{2.74}$ \\
\hline & $\mathrm{C} 1-\mathrm{C} 6$ & $0.7134 \mathrm{sp}^{1.47}+0.7008 \mathrm{sp}^{1.92}$ & $0.7008 \mathrm{sp}^{1.47}-0.7134 \mathrm{sp}^{1.92}$ & $0.7132 \mathrm{sp}^{1.47}+0.7010 \mathrm{sp}^{1.91}$ & $0.7010 \mathrm{sp}^{1.47}-0.7132 \mathrm{sp}^{1.91}$ & $0.7136 \mathrm{sp}^{1.47}+0.7006 \mathrm{sp}^{1.92}$ & $0.7006 \mathrm{sp}^{1.47}-0.7136 \mathrm{sp}^{1.92}$ \\
\hline & $\mathrm{C} 2-\mathrm{Cg}$ & $0.7150 \mathrm{sp}^{1.46}+0.6991 \mathrm{sp}^{1.94}$ & $0.6991 \mathrm{sp}^{1.46}-0.7150 \mathrm{sp}^{1.94}$ & $0.7149 \mathrm{sp}^{1.45}+0.6992 \mathrm{sp}^{1.93}$ & $0.6992 \mathrm{sp}^{1.45}-0.7149 \mathrm{sp}^{1.93}$ & $0.7152 \mathrm{sp}^{1.46}+0.6990 \mathrm{sp}^{1.94}$ & $0.6990 \mathrm{sp}^{1.46}-0.7152 \mathrm{sp}^{1.94}$ \\
\hline \multirow{3}{*}{ 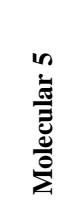 } & C1- N5 & $0.6525 \mathrm{sp}^{2.62}+0.7578 \mathrm{sp}^{3.48}$ & $0.7578 \mathrm{sp}^{2.62}-0.6525 \mathrm{sp}^{3.48}$ & $0.6525 \mathrm{sp}^{2.62}+0.7578 \mathrm{sp}^{3.48}$ & $0.7578 \mathrm{sp}^{2.62}-0.6525 \mathrm{sp}^{3.48}$ & $0.6531 \mathrm{sp}^{2.63}+0.7573 \mathrm{sp}^{3.50}$ & $0.7573 \mathrm{sp}^{2.63}-0.6531 \mathrm{sp}^{3.50}$ \\
\hline & $\mathrm{C} 2-\mathrm{N} 3$ & $0.6341 \mathrm{sp}^{2.81}+0.7733 \mathrm{sp}^{2.58}$ & $0.7733 \mathrm{sp}^{2.81}-0.6341 \mathrm{sp}^{2.58}$ & $0.6341 \mathrm{sp}^{2.81}+0.7733 \mathrm{sp}^{2.58}$ & $0.7733 \mathrm{sp}^{2.81}-0.6341 \mathrm{sp}^{2.58}$ & $0.6345 \mathrm{sp}^{2.81}+0.7729 \mathrm{sp}^{2.59}$ & $0.7729 \mathrm{sp}^{2.81}-0.6345 \mathrm{sp}^{2.59}$ \\
\hline & $\mathrm{C} 4-\mathrm{N} 5$ & $0.6322 \mathrm{sp}^{1.82}+0.7748 \mathrm{sp}^{1.32}$ & $0.7748 \mathrm{sp}^{1.82}-0.6322 \mathrm{sp}^{1.32}$ & $0.6322 \mathrm{sp}^{1.82}+0.7748 \mathrm{sp}^{1.32}$ & $0.7748 \mathrm{sp}^{1.82}-0.6322 \mathrm{sp}^{1.32}$ & $0.6327 \mathrm{sp}^{1.82}+0.7744 \mathrm{sp}^{1.33}$ & $0.7744 \mathrm{sp}^{1.82}-0.6327 \mathrm{sp}^{1.33}$ \\
\hline
\end{tabular}


Table 3b. Contd.

\begin{tabular}{|c|c|c|c|c|c|c|}
\hline C4-N5 & $0.6593 \mathrm{sp}+0.7519 \mathrm{sp}^{99.99}$ & $0.7519 \mathrm{sp}-0.6593 \mathrm{sp} \mathrm{p}^{99.99}$ & $0.6593 \mathrm{sp}+0.7519 \mathrm{sp}^{99.99}$ & $0.7519 \mathrm{sp}-0.6593 \mathrm{sp}^{99.99}$ & $0.6584 \mathrm{sp}+0.7527 \mathrm{sp}^{99.99}$ & $0.7527 \mathrm{sp}-0.6584 \mathrm{sp}^{99.99}$ \\
\hline $\mathrm{N} 3-\mathrm{C} 4$ & $0.7909 \mathrm{sp}^{2.64}+0.6122 \mathrm{sp}^{2.77}$ & $0.6122 \mathrm{sp}^{2.64}-0.7907 \mathrm{sp}^{2.77}$ & $0.7909 \mathrm{sp}^{2.64}+0.6122 \mathrm{sp}^{2.77}$ & $0.6122 \mathrm{sp}^{2.64}-0.7907 \mathrm{sp}^{2.77}$ & $0.7907 \mathrm{sp}^{2.66}+0.6122 \mathrm{sp}^{2.79}$ & $0.6122 \mathrm{sp}^{2.66}-0.7907 \mathrm{sp}^{2.79}$ \\
\hline
\end{tabular}

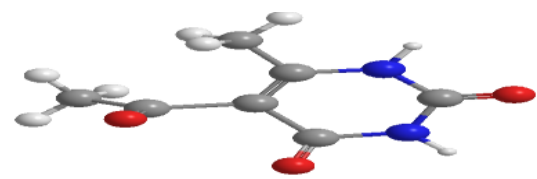

(a)

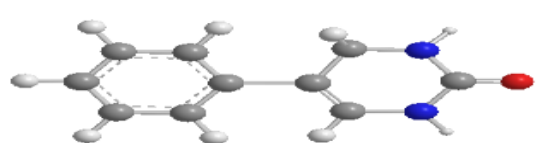

(b)
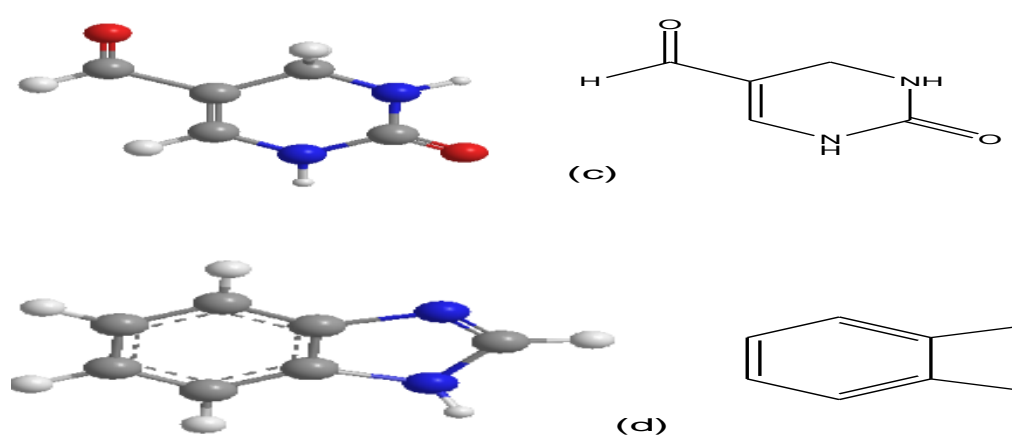

(d)

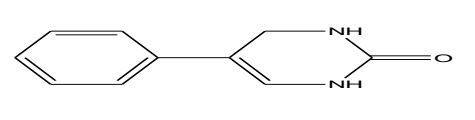

(c)
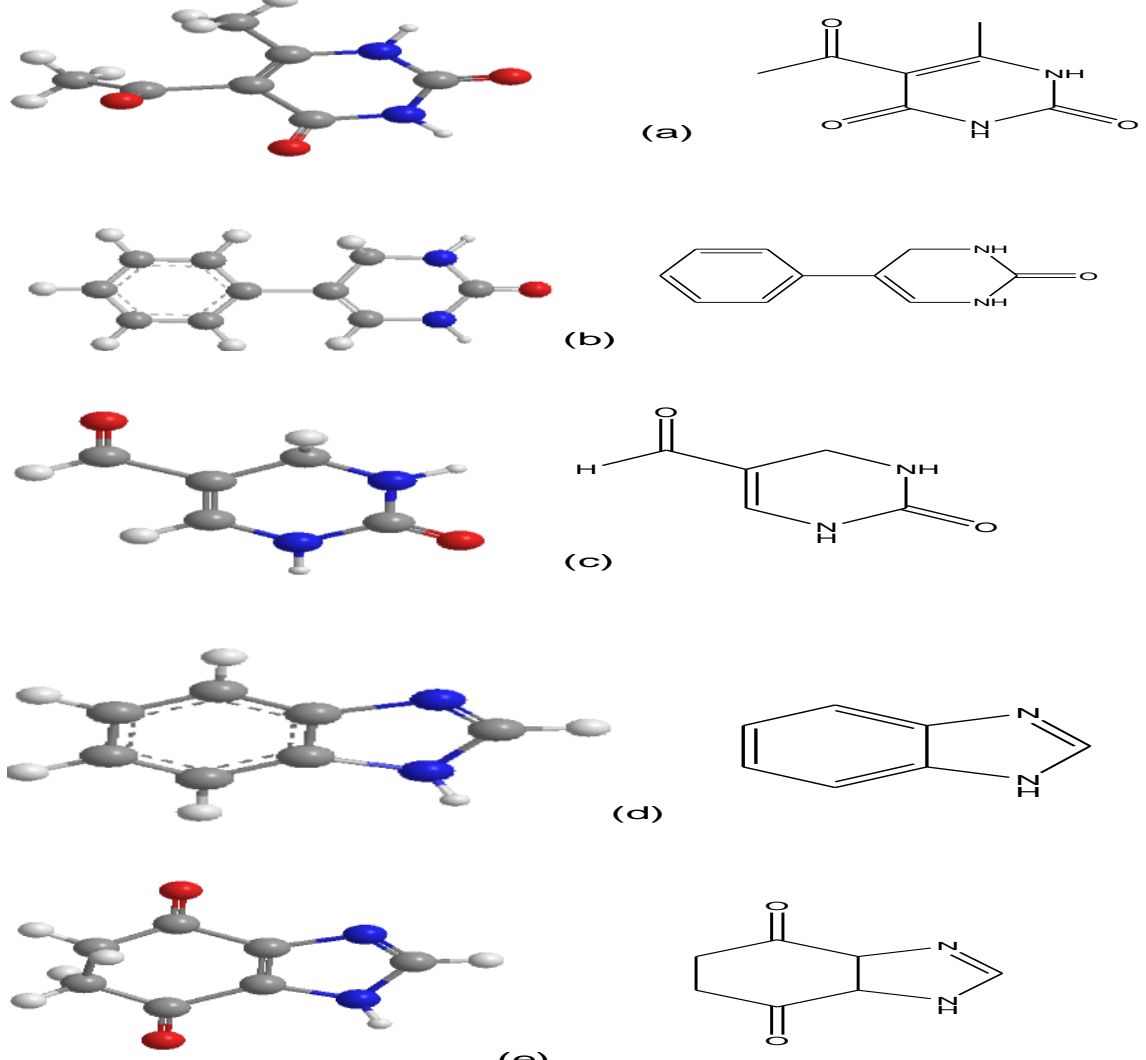

Figure 1. Structures of Molecules 1, 2, 3, 4 and 5 shown as chemical structures and energy-minimized structures (ball-and-stick model). (a) Molecule 1. 5-acetyl-6methylpyrimidine-2,4(1H,3H)- dione. (b) Molecule 2. 5-acetyl-6-methylpyrimidine2,4 $(1 \mathrm{H}, 3 \mathrm{H})$ - dione. (c) Molecule 3. 1,2,3,4-tetrahydro-2-oxopyrimidine-5-carbaldehyde. (d) Molecule 4. 1H-benzo[d]imidazole. (5) Molecule 5. 5,6-dihydro-1Hbenzo[d]imidazole-4,7-dione. 


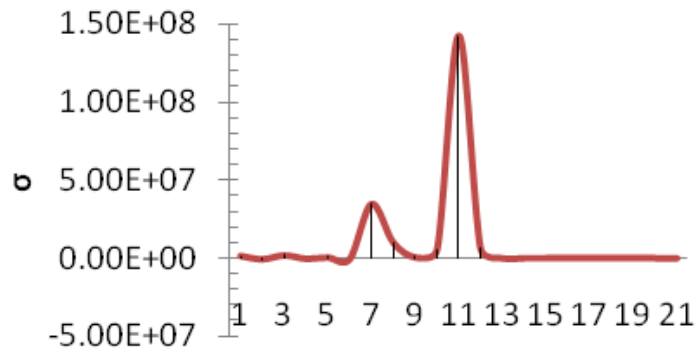

Atoms

Molecule 1 ( HF/6-31+G)

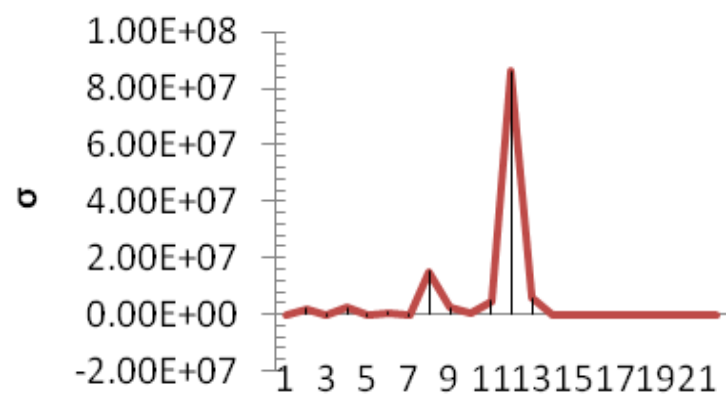

Atoms

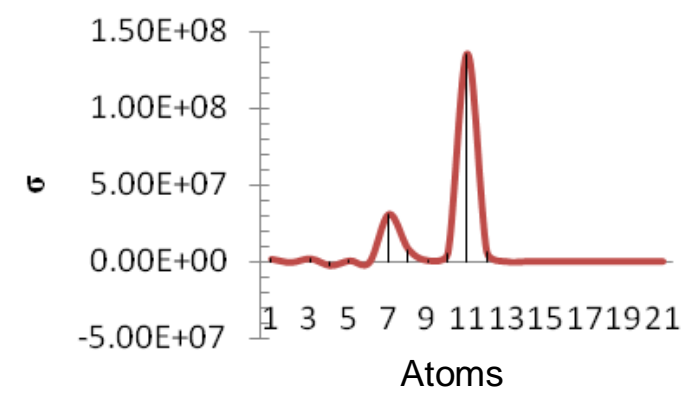

Molecule 1 ( HF/6-31G)

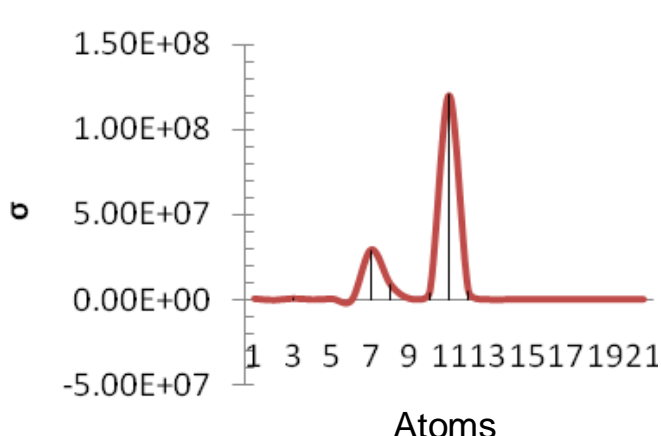

Molecule 1 ( B3LYP/6-31G)

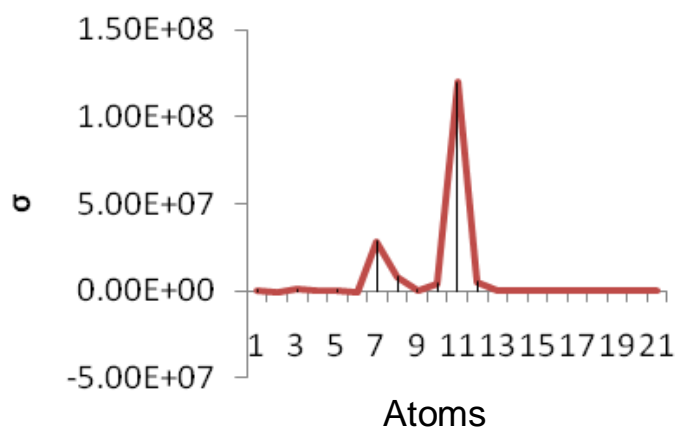

Molecule 1 ( B3PW91/6-31G)

Molecule 1 ( BLYP/6-31G)

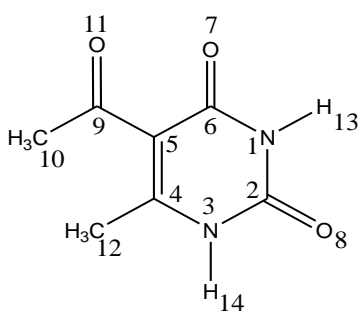

Molecule 1

Figure 2. Diagrams which show chemical shifts for each of the atom at the Molecule (1). 


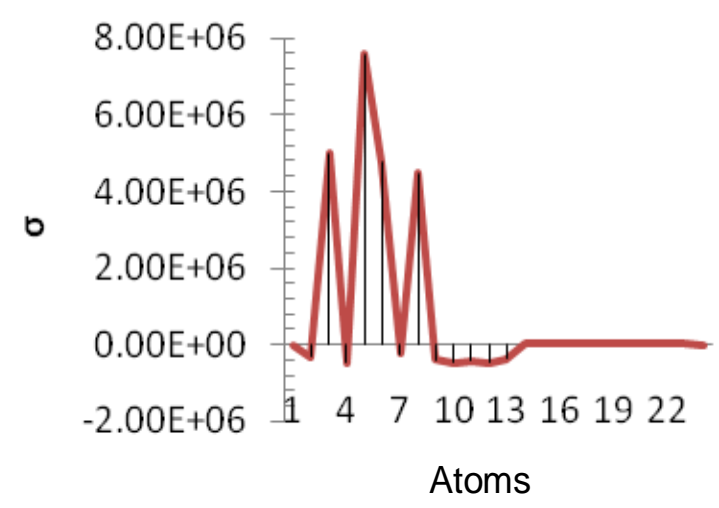

Molecule 2 ( HF/6-31G)

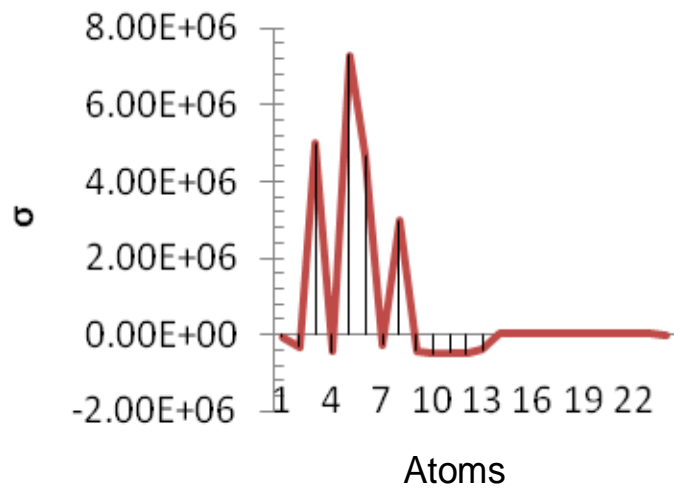

Molecule 2 ( HF/6-31+G)

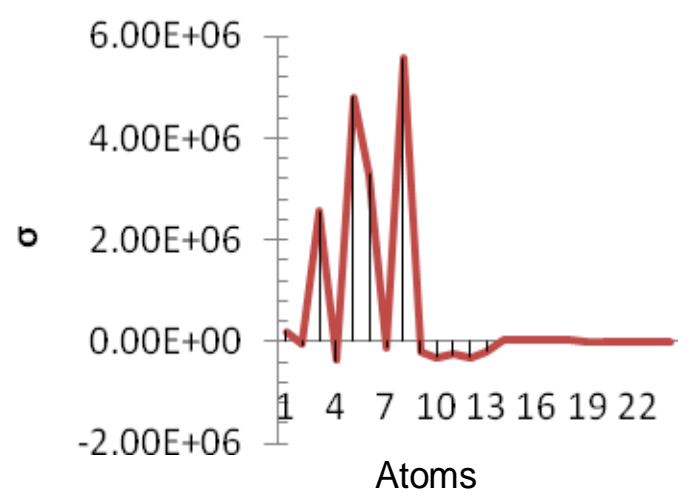

Molecule 2 ( B3LYP/6-31G)

Molecule 2 ( HF/6-31G*)

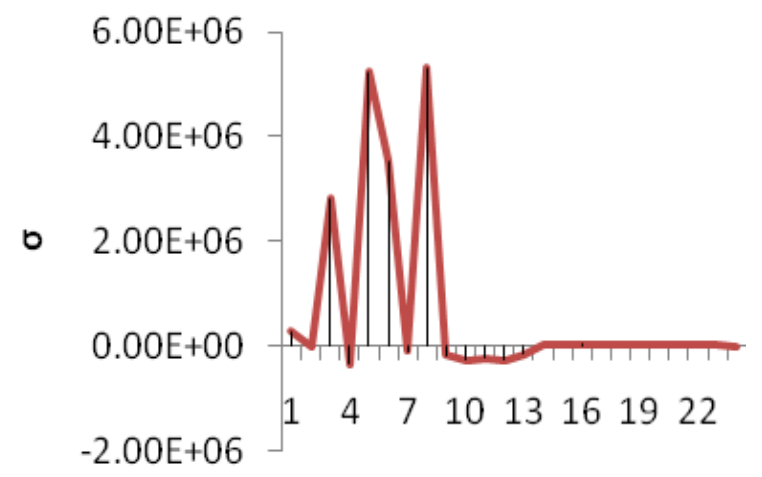

Atoms

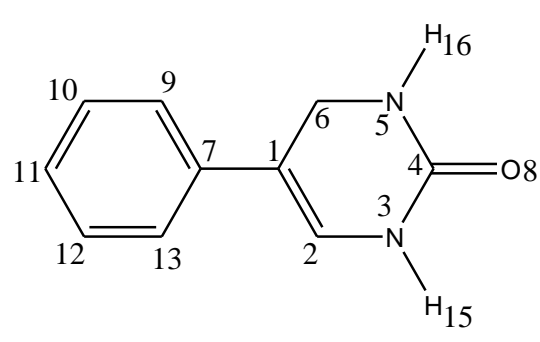

Molecule 2

\section{Molecule 2 ( B3LYP/6-31G)}

Figure 3. Diagrams which show chemical shifts for each of the atom at the Molecule (2). 


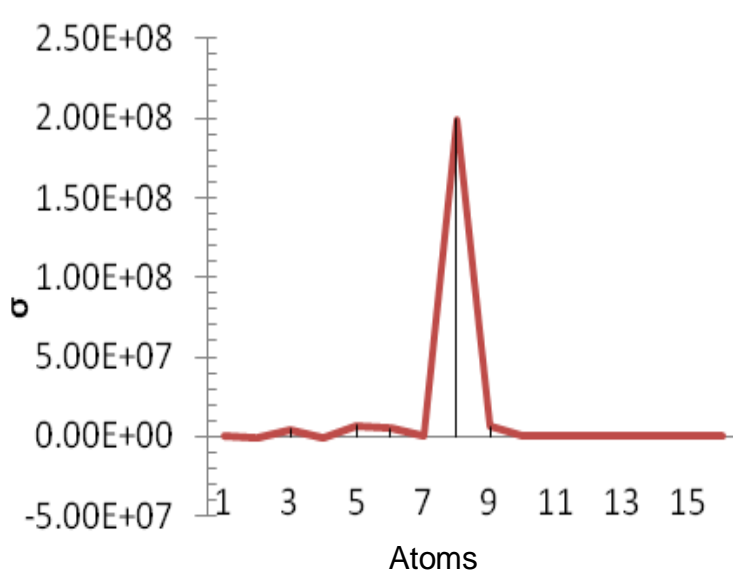

Molecule 3 ( HF/6-31G)

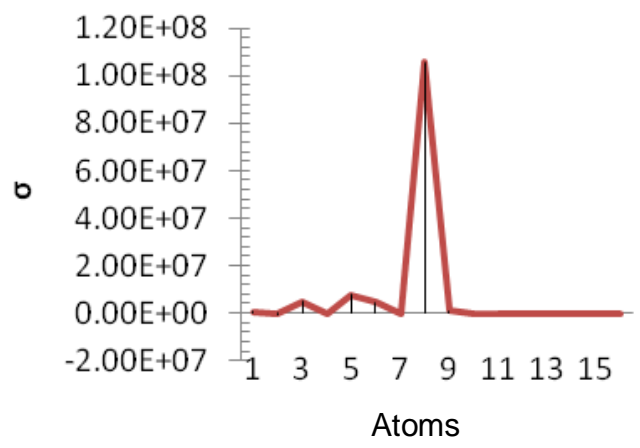

Molecule 3 ( HF/6-31G*)

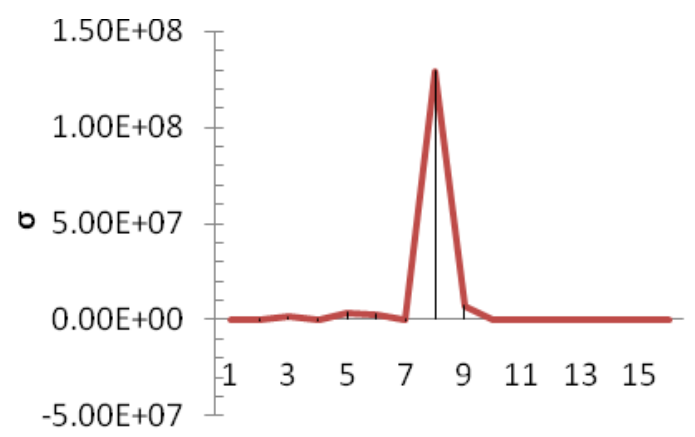

Atoms

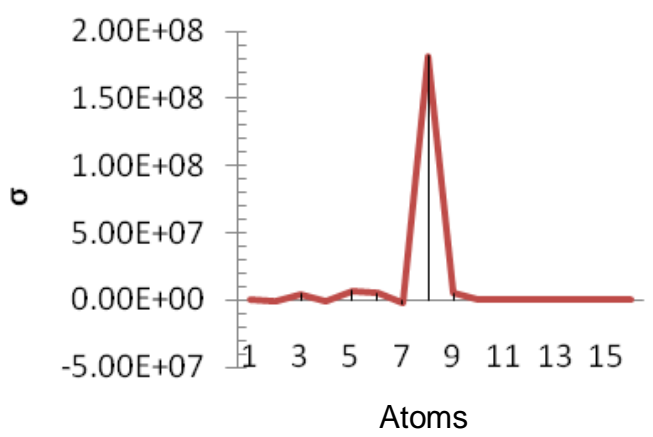

Molecule 3 ( HF/6-31+G)

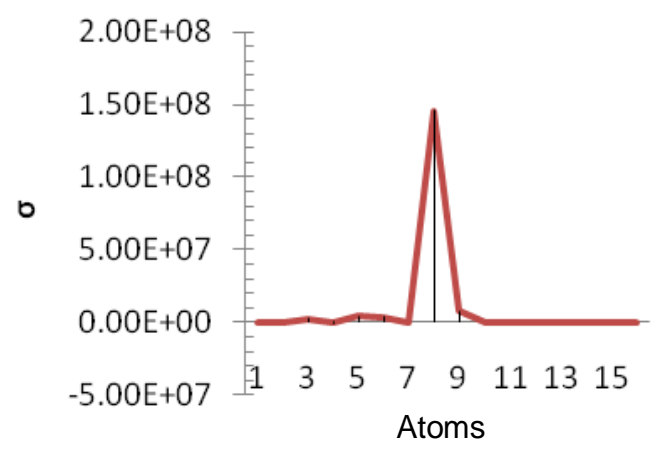

Molecule 3 ( B3LYP/6-31G)

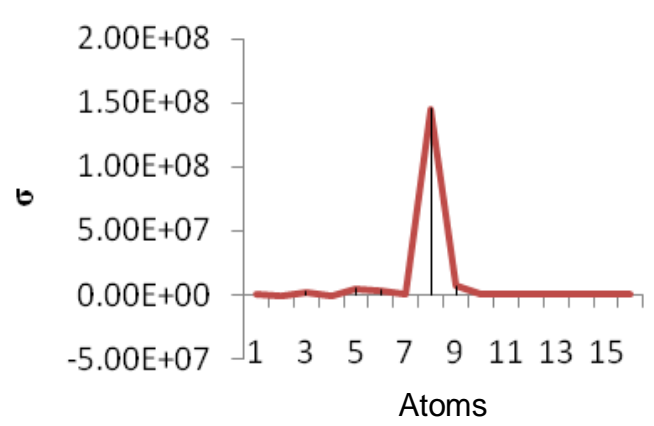

Molecule 3 ( B3PW91/6-31G)

Molecule 3 ( BLYP/6-31G)<smiles>CN1C=C(C(=O)S)CNC1=O</smiles>

\section{Molecule 3}

Figure 4. Diagrams which show chemical shifts for each of the atom at the Molecule (3). 


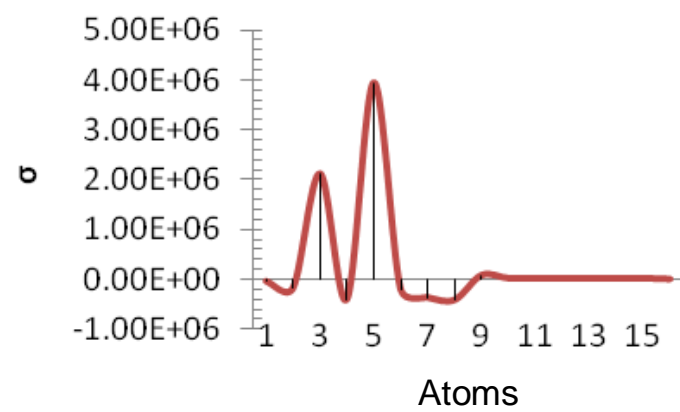

Molecule 4 ( HF/6-31G)
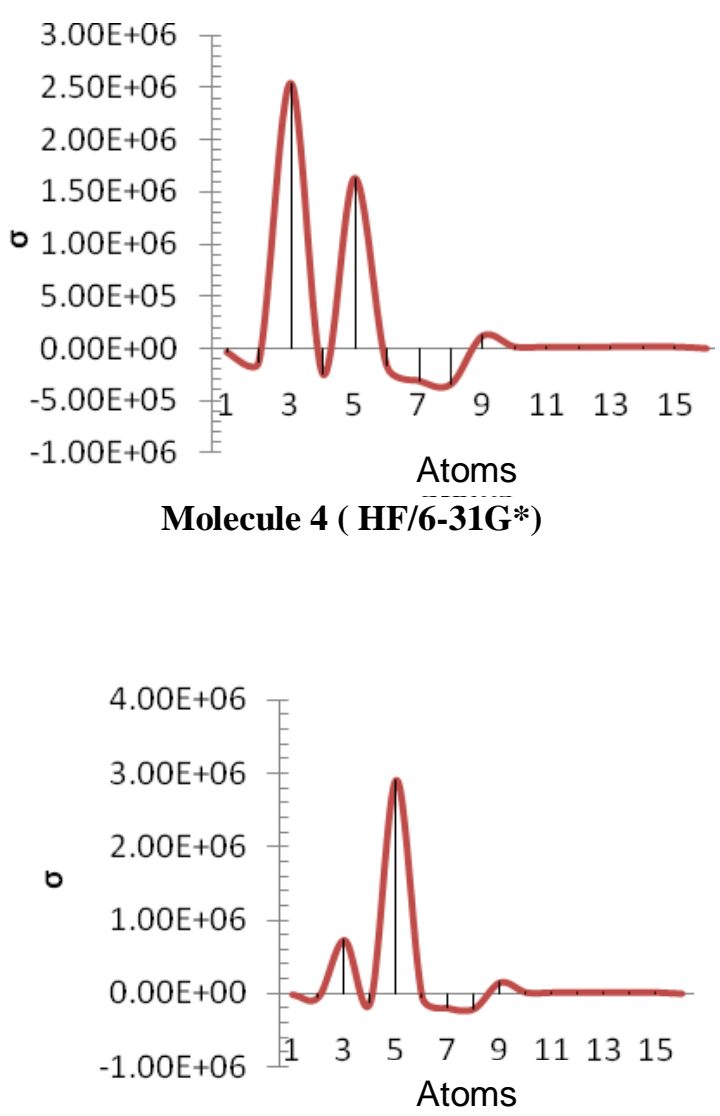

Molecule 4 ( BLYP/6-31G)

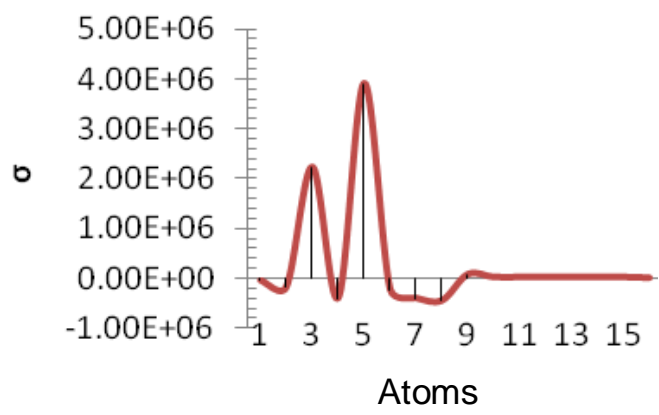

Molecule 4 ( HF/6-31+G)

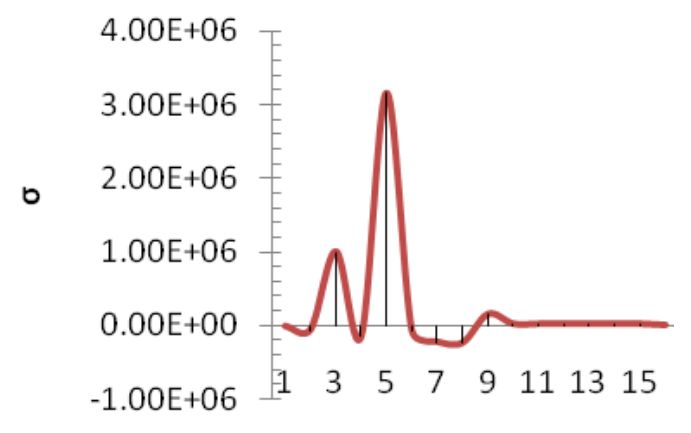

Atoms

Molecule 4 ( B3LYP/6-31G)

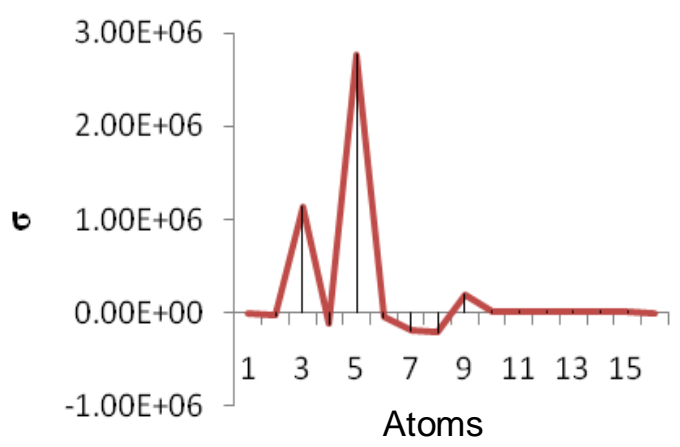

Molecule 4 ( B3PW91/6-31G)

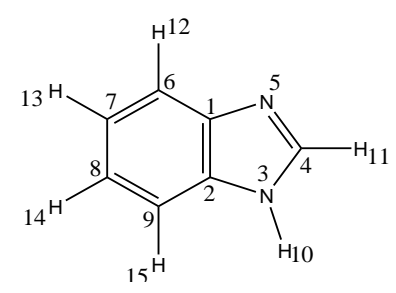

Molecule 4

Figure 5. Diagrams which show chemical shifts for each of the atom at the Molecule (4). 


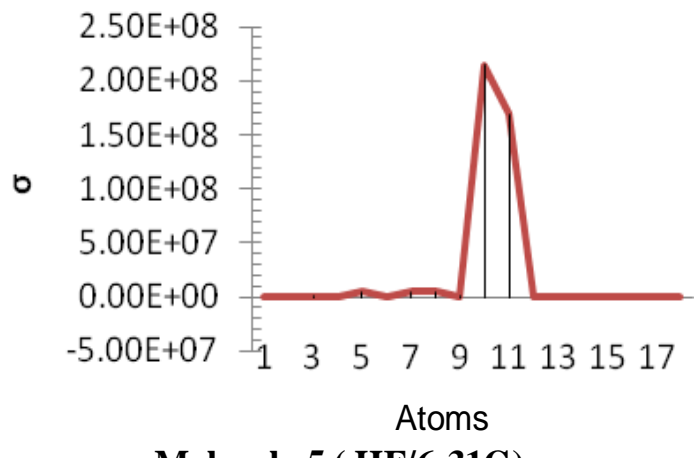

Molecule 5 ( HF/6-31G)
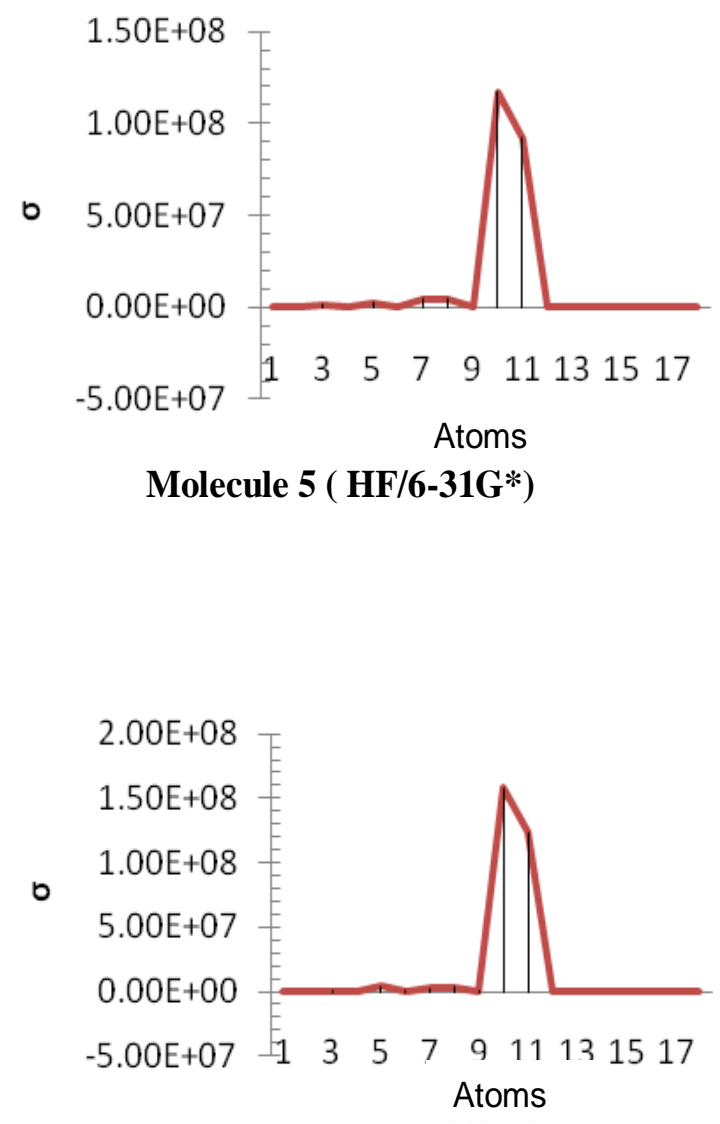

Molecule 5 ( BLYP/6-31G)
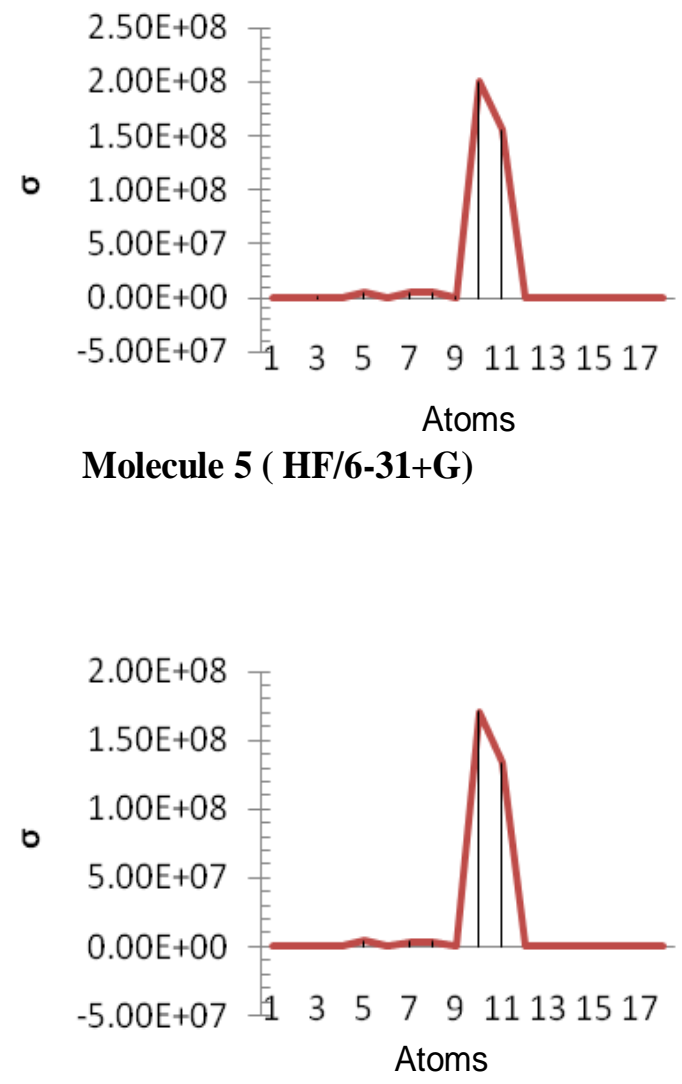

Molecule 5 ( B3LYP/6-31G)

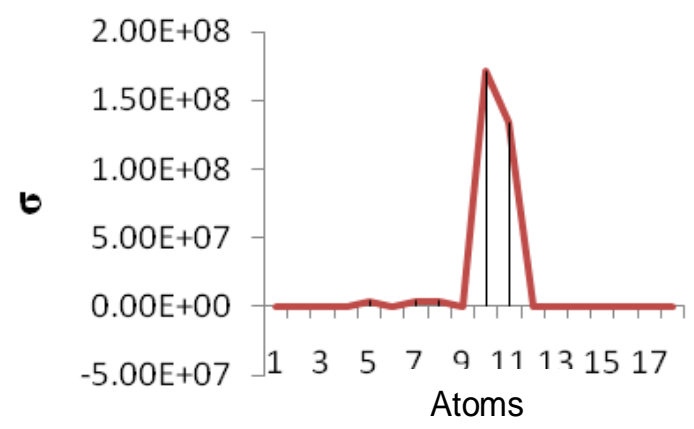

Molecule 5 ( B3PW91/6-31G)<smiles>Cc1nc2c(n1C)C(=O)CCC2=O</smiles>

\section{Molecule 5}

Figure 6. Diagrams which show chemical shifts for each of the atom at the Molecule (5). 


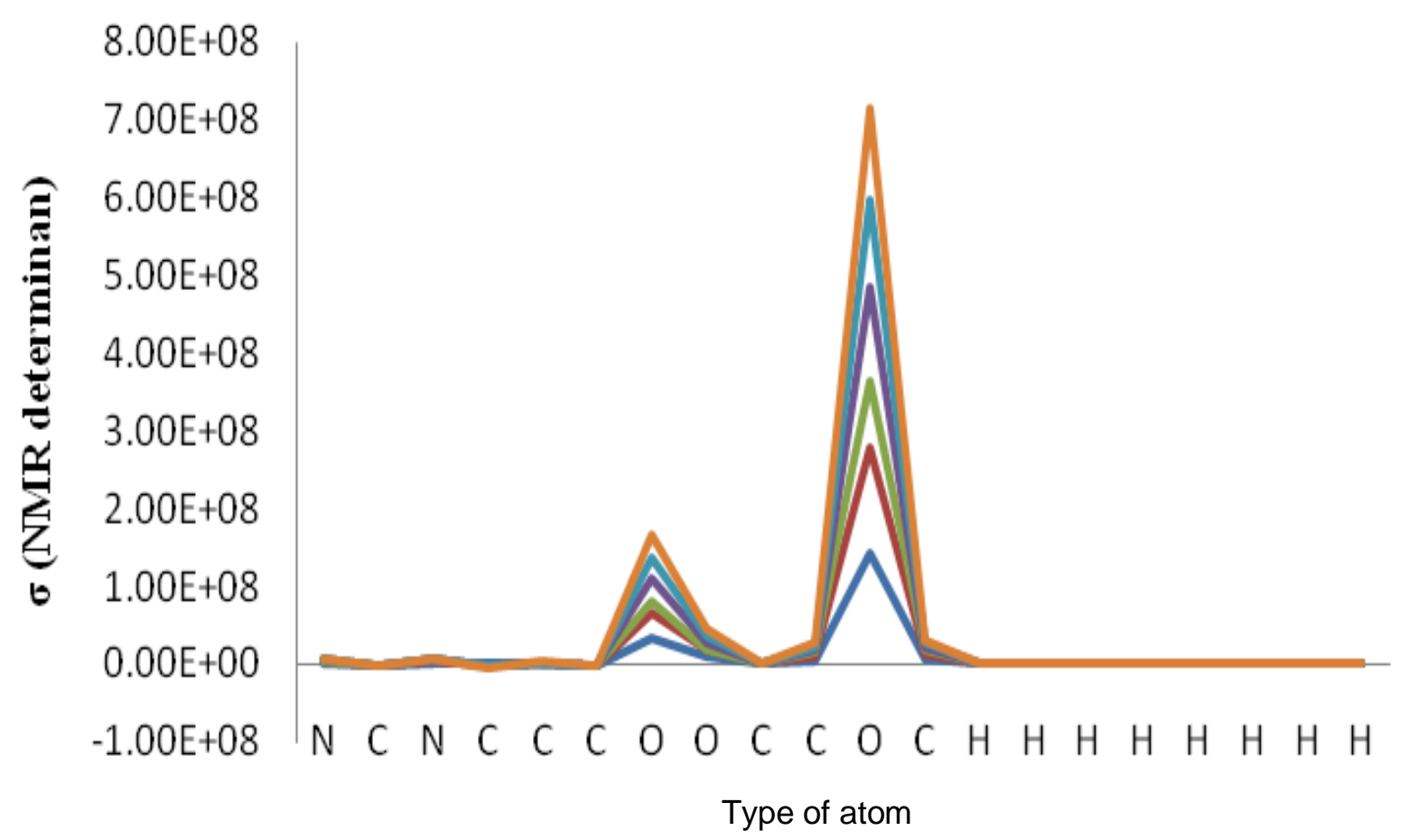

$\longrightarrow$ Series $1-$ Series $2 \longrightarrow$ Series $3 \longrightarrow$ Series $4 \longrightarrow$ Series $5 \longrightarrow$ Series 6

Figure 7. The graphs of chemical shifts for Molecule 1. (Series1) HF/6-31 g, (Series 2) HF/6-31+g (Series 3) HF/6$31 \mathrm{~g}^{*}$,(Series4) B3LYP/6-31 g, (Series 5) BLYP/6-31 g, (Series 6) B3PW91/6-31 g.

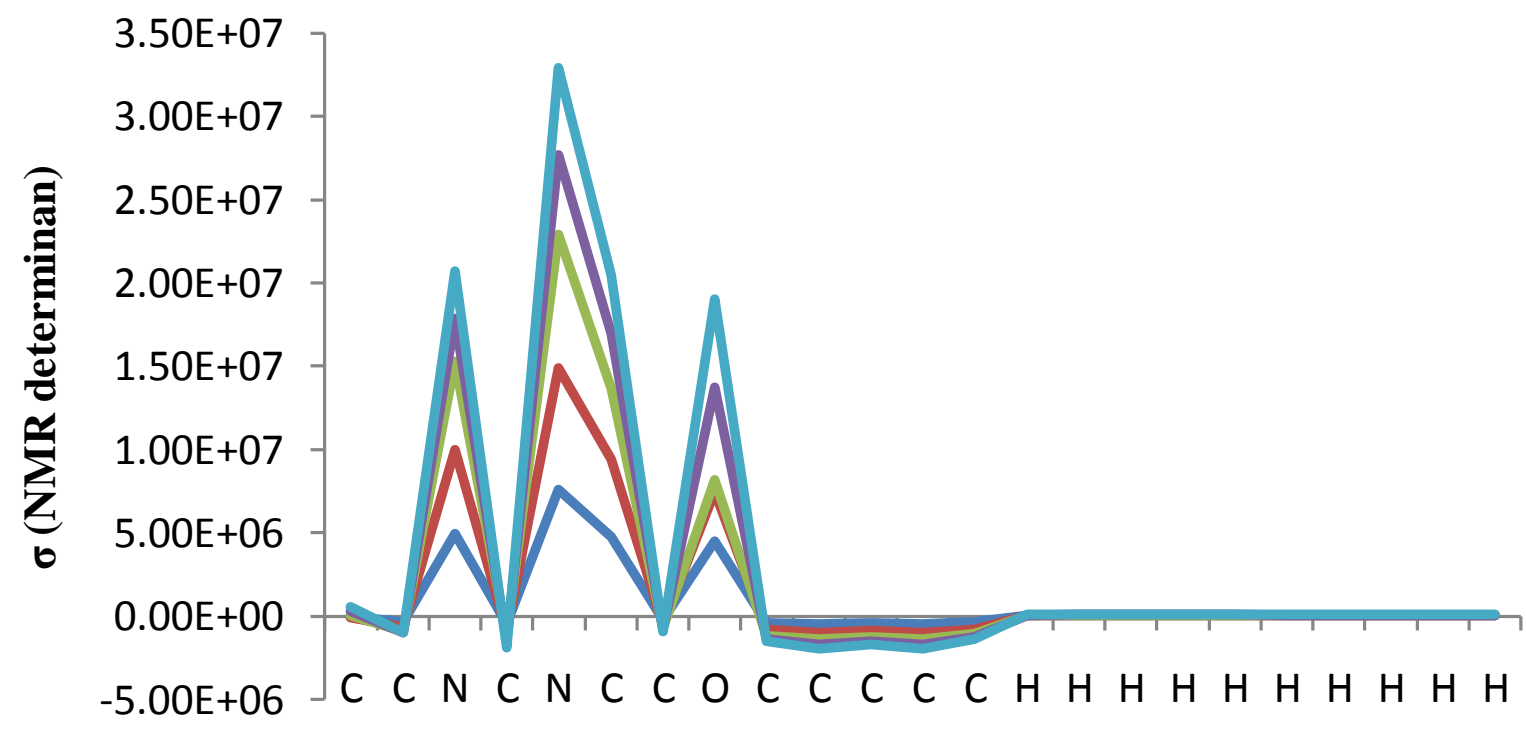
Type of atom

- Series1 - Series2 Series3 Series4 Series5

Figure 8. The graphs of chemical shifts for Molecule 2.(Series 1) HF/6-31g, (Series 2) HF/6-31+g (Series 3) HF/6$31 \mathrm{~g}^{*}$, (Series 4) B3LYP/6-31 g , (Series 5) B3PW91/6-31 g. 


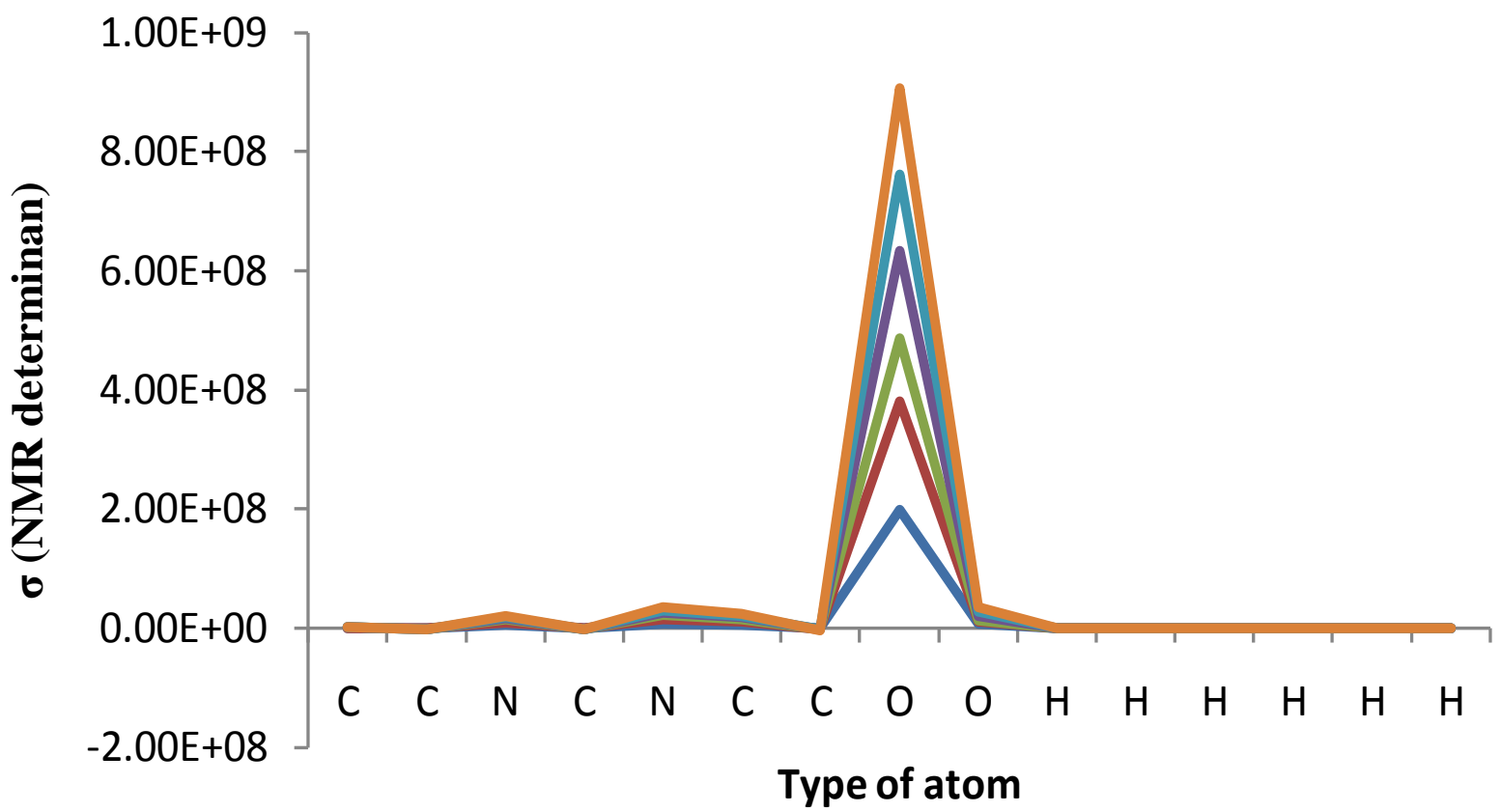

$\longrightarrow$ Series $1 \longrightarrow$ Series $2 \longrightarrow$ Series3 $\longrightarrow$ Series $4 \longrightarrow$ Series5 $\longrightarrow$ Series6

Figure 9. The graphs of chemical shifts for Molecule 3.(Series 1) HF/6-31 g, (Series 2) HF/6-31+g (Series 3) HF/6-31 $\mathrm{g}^{*}$, (Series 4) B3LYP/6-31 g, (Series 5) BLYP/6-31g, (Series 6) B3PW91/6-31 g.

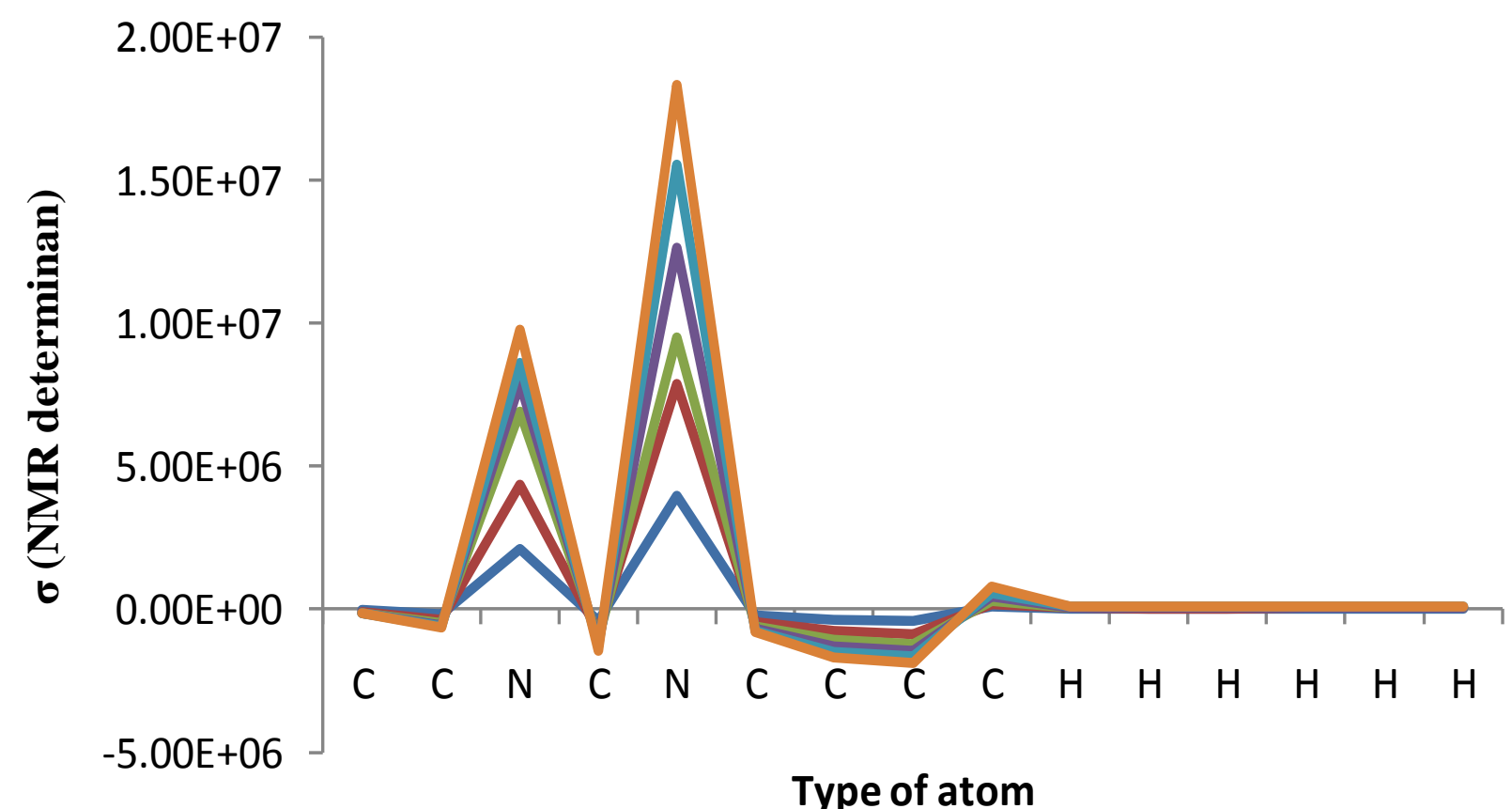

\section{$\longrightarrow$ Series1 - Series2 - Series3 $\longrightarrow$ Series4 $\longrightarrow$ Series5}

Figure 10. The graphs of chemical shifts for Molecule 4.(Series 1) HF/6-31 g , (Series 2) HF/6-31+g (Series 3) HF/6-31 $\mathrm{g}^{\star}$, (Series 4) B3LYP/6-31 g, (Series 5) BLYP/6-31 g, (Series 6) B3PW91/6-31 g. 
Table 4. Ralative GIAO Magnetic shielding for active site of studied moleculars obtained using different methods.

\begin{tabular}{|c|c|c|c|c|c|c|c|c|c|c|c|c|c|}
\hline \multirow{2}{*}{\multicolumn{2}{|c|}{$\begin{array}{c}\text { Method } \\
\text { Basis set }\end{array}$}} & \multicolumn{6}{|c|}{ HF } & \multicolumn{2}{|c|}{ B3LYP } & \multicolumn{2}{|c|}{ BLYP } & \multicolumn{2}{|c|}{ B3PW91 } \\
\hline & & \multicolumn{2}{|c|}{$6-31 G$} & \multicolumn{2}{|c|}{$6-31 G^{\star}$} & \multicolumn{2}{|c|}{$6-31+G$} & \multicolumn{6}{|c|}{$6-31 G$} \\
\hline 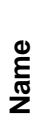 & Atoms & $\begin{array}{c}\text { Shielding } \\
\text { (ppm) }\end{array}$ & 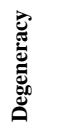 & $\begin{array}{c}\text { Shielding } \\
\text { (ppm) }\end{array}$ & 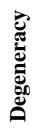 & $\begin{array}{c}\text { Shielding } \\
\text { (ppm) }\end{array}$ & 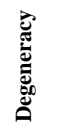 & $\begin{array}{c}\text { Shielding } \\
\text { (ppm) }\end{array}$ & 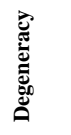 & $\begin{array}{c}\text { Shieldin } \\
\text { (ppm) }\end{array}$ & 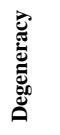 & $\begin{array}{c}\text { Shielding } \\
\text { (ppm) }\end{array}$ & 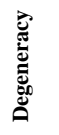 \\
\hline \multirow{9}{*}{ 冚 } & $\mathrm{N}(1)$ & 127.7 & 1 & - & - & - & - & - & - & - & - & - & - \\
\hline & $\mathrm{N}(3)$ & 137.4 & 1 & - & - & - & - & - & - & - & - & - & - \\
\hline & $H(13)$ & 26.95 & 1 & - & - & - & - & - & - & - & - & - & - \\
\hline & $\mathrm{H}(14)$ & 27.72 & 1 & - & - & - & - & - & - & - & - & - & - \\
\hline & $C(2)$ & 50.7 & 1 & - & - & - & - & - & - & - & - & - & - \\
\hline & $\mathrm{C}(4)$ & 35.5 & 1 & - & - & - & - & - & - & - & - & - & - \\
\hline & $C(6)$ & 33.5 & 1 & - & - & - & - & - & - & - & - & - & - \\
\hline & $\mathrm{C}(9)$ & -5.5 & 1 & - & - & - & - & - & - & - & - & - & - \\
\hline & $\mathrm{O}(8)$ & -2.3 & 1 & - & - & - & - & - & - & - & - & - & - \\
\hline \multirow{9}{*}{ 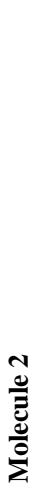 } & $\mathrm{N}(3)$ & 179 & 1 & 179.5 & 1 & 179.2 & 1 & 146.2 & 1 & - & - & 150.3 & 1 \\
\hline & $\mathrm{N}(5)$ & 205.3 & 1 & 206.1 & 1 & 203.2 & 1 & 177.2 & 1 & - & - & 182.2 & 1 \\
\hline & $H(15)$ & 29.26 & 1 & 28.78 & 1 & 28.81 & 1 & 28.31 & 1 & - & - & 28.15 & 1 \\
\hline & $\mathrm{H}(16)$ & 30.7 & 1 & 30.09 & 1 & 29.7 & 1 & 29.77 & 1 & - & - & 29.67 & 1 \\
\hline & $\mathrm{H}(17)$ & 29.31 & 2 & 28.73 & 1 & 29.07 & 1 & 28.13 & 1 & - & - & 28.07 & 1 \\
\hline & $C(1)$ & 96.3 & 1 & 96.54 & 1 & 95 & 1 & 89.57 & 1 & - & - & 93.2 & 1 \\
\hline & $C(2)$ & 82.3 & 1 & 78.06 & 1 & 82.2 & 1 & 77.56 & 1 & - & - & 80.31 & 1 \\
\hline & $C(4)$ & 47.06 & 1 & 55.87 & 1 & 45.02 & 1 & 49.3 & 1 & - & - & 53.03 & 1 \\
\hline & $\mathrm{O}(8)$ & 34.8 & 1 & 84.77 & 1 & 34.8 & 1 & 4.45 & 1 & - & - & 10.17 & 1 \\
\hline
\end{tabular}


Table 4. Contd.

\begin{tabular}{|c|c|c|c|c|c|c|c|c|c|c|c|c|c|}
\hline \multirow{9}{*}{ 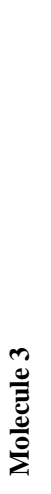 } & $N(3)$ & 173 & 1 & 175 & 1 & 173.2 & 1 & 139.4 & 1 & 126.96 & 1 & 143.89 & 1 \\
\hline & $N(5)$ & 203.5 & 1 & 205.5 & 1 & 201.4 & 1 & 173.34 & 1 & 161.11 & 1 & 178.1 & 1 \\
\hline & $H(11)$ & 28.94 & 1 & 28.57 & 1 & 28.39 & 1 & 28.06 & 1 & 27.82 & 1 & 27.92 & 1 \\
\hline & $H(12)$ & 30.94 & 1 & 29.93 & 1 & 29.65 & 1 & 29.47 & 1 & 29.18 & 1 & 29.38 & 1 \\
\hline & $H(15)$ & 23.17 & 1 & 23.76 & 1 & 23.08 & 1 & 22.8 & 1 & 22.63 & 1 & 22.71 & 1 \\
\hline & $C(4)$ & 46.8 & 1 & 58.1 & 1 & 46.1 & 1 & 49.64 & 1 & 48.83 & 1 & 53.39 & 1 \\
\hline & $C(6)$ & 172.2 & 1 & 165.61 & 1 & 172 & 1 & 151.99 & 1 & 143.13 & 1 & 155.58 & 1 \\
\hline & $C(7)$ & 9 & 1 & 27 & 1 & 8.6 & 1 & 10.73 & 1 & 11.3 & 1 & 13.2 & 1 \\
\hline & O(9) & 19 & 1 & 75 & 1 & 26.58 & 1 & -13.26 & 1 & -23.36 & 1 & -7.59 & 1 \\
\hline \multirow{9}{*}{ 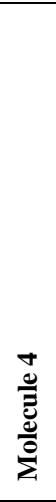 } & $N(3)$ & 142 & 1 & 147.61 & 1 & 143.88 & 1 & 117.1 & 1 & 108.35 & 1 & 120.57 & 1 \\
\hline & $N(5)$ & -19.29 & 1 & 20 & 1 & -19.43 & 1 & -20.67 & 1 & -22.68 & 1 & -14.6 & 1 \\
\hline & $\mathrm{H}(10)$ & 26.59 & 1 & 25.73 & 1 & 26.26 & 1 & 25.41 & 1 & 25.08 & 1 & 25.3 & 1 \\
\hline & $H(11)$ & 25.29 & 1 & 24.98 & 1 & 25.18 & 1 & 25.29 & 1 & 25.23 & 1 & 25.17 & 1 \\
\hline & $\mathrm{H}(14)$ & 25.81 & 1 & 25.36 & 1 & 25.63 & 1 & 25.55 & 2 & 25.47 & 2 & 25.42 & 2 \\
\hline & $H(15)$ & 25.95 & 2 & 25.45 & 2 & 25.73 & 2 & 25.6 & 3 & 25.48 & 3 & 25.47 & 3 \\
\hline & $C(1)$ & 62.8 & 1 & 63.19 & 1 & 61.39 & 1 & 53.97 & 1 & 50.13 & 1 & 57.69 & 1 \\
\hline & $\mathrm{C}(2)$ & 72.09 & 1 & 72.5 & 1 & 71.4 & 1 & 65.5 & 1 & 62.09 & 1 & 69.25 & 1 \\
\hline & $\mathrm{C}(4)$ & 60.7 & 1 & 63.55 & 1 & 59.25 & 1 & 59.89 & 1 & 58 & 1 & 62.86 & 1 \\
\hline \multirow{9}{*}{ 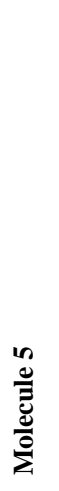 } & $N(3)$ & 123.66 & 1 & 131.33 & 1 & 126.45 & 1 & 105.9 & 1 & 99.58 & 1 & 109.49 & 1 \\
\hline & $\mathrm{N}(5)$ & -39 & 1 & 2.26 & 1 & -37.8 & 1 & -44.8 & 1 & -48.6 & 1 & -38.82 & 1 \\
\hline & $H(12)$ & 25.38 & 1 & 24.89 & 1 & 25.1 & 1 & 24.45 & 1 & 24.2 & 1 & 24.4 & 1 \\
\hline & $\mathrm{H}(13)$ & 25.21 & 1 & 25.06 & 1 & 25.19 & 1 & 25.18 & 1 & 25.12 & 1 & 25.07 & 1 \\
\hline & $C(1)$ & 59.55 & 1 & 58.7 & 1 & 59.28 & 1 & 52.39 & 1 & 49.79 & 1 & 55.94 & 1 \\
\hline & $\mathrm{C}(2)$ & 70.31 & 1 & 70.5 & 1 & 71 & 1 & 61.9 & 1 & 58.7 & 1 & 65.39 & 1 \\
\hline & $C(4)$ & 61.56 & 1 & 65.12 & 1 & 59.92 & 1 & 58.67 & 1 & 55.92 & 1 & 61.72 & 1 \\
\hline & $C(6)$ & 83 & 1 & 79.67 & 1 & 80.15 & 1 & 75.99 & 1 & 73.79 & 1 & 78.63 & 1 \\
\hline & $\mathrm{C}(9)$ & 94.84 & 1 & 93.94 & 1 & 95 & 1 & 88.81 & 1 & 86.1 & 1 & 91.5 & 1 \\
\hline
\end{tabular}




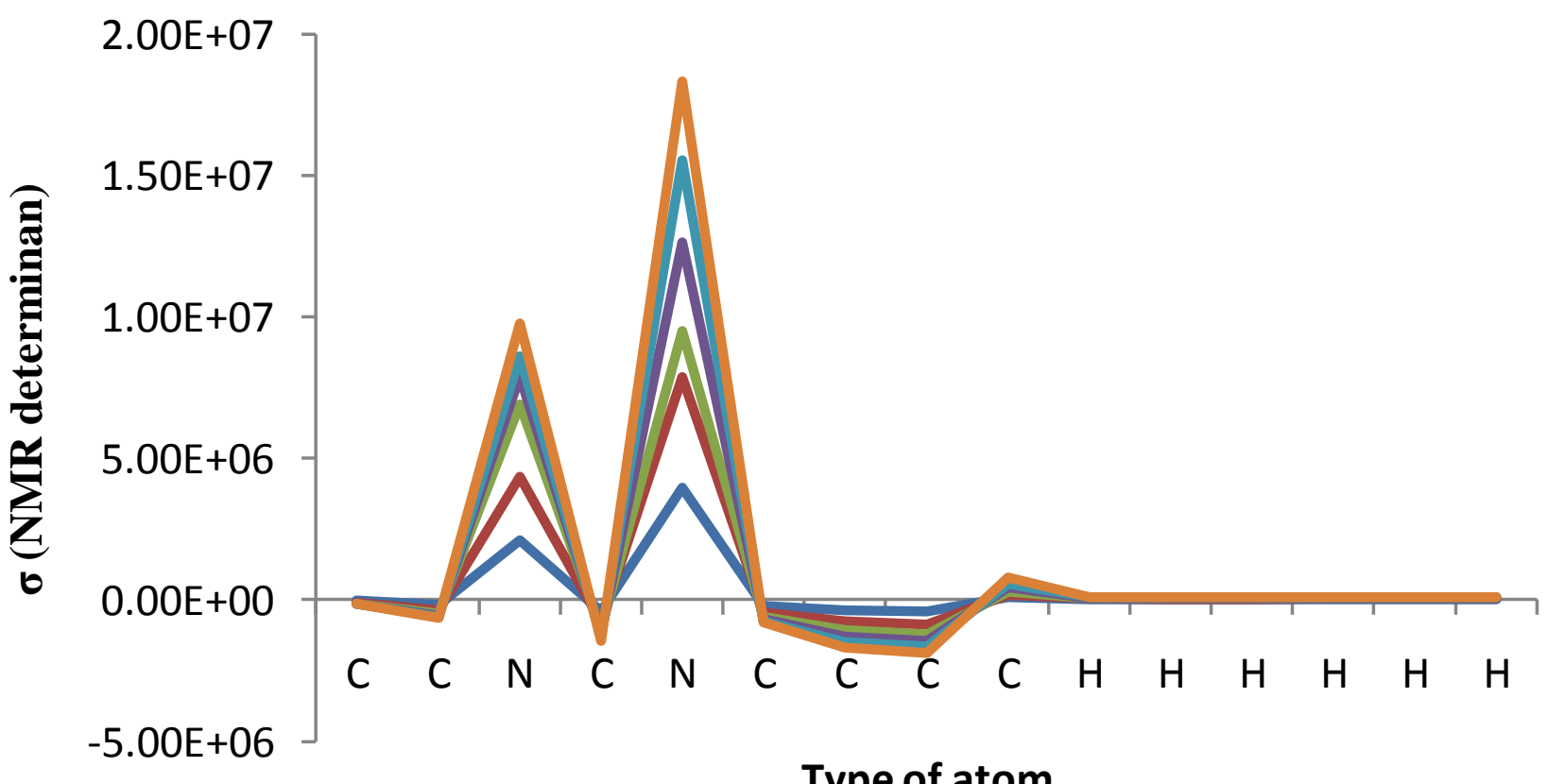

Type of atom

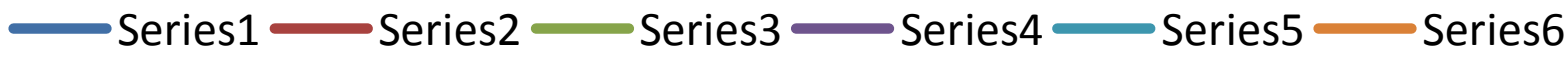

Figure 11. The graphs of chemical shifts for Molecule 5.(Series 1) HF/6-31 g, (Series 2) HF/6-31+g (Series 3) HF/6-31 $\mathrm{g}^{*}$, (Series 4) B3LYP/6-31 g, (Series 5) BLYP/6-31g , (Series 6) B3PW91/6-31 g.

Table 4 shows of GIAO magnetic shielding for some of atoms. As shown in Table 4, in Molecule 1, just HF/6-31G response. In Molecule 1, at HF/6-31G, all atoms degeneracy value is same, and $\mathrm{N}_{1}, \mathrm{~N}_{3}$ atoms contain positive shielding values, while $\mathrm{C}_{9}$ and $\mathrm{O}_{8}$ atoms contain negative shielding values. In Molecule 2, in all of the methods, except BLYP/6-31G level, $\mathrm{N}_{3}$ and $\mathrm{N}_{5}$ atoms have the greatest positive shielding values, at $\mathrm{HF} / 6-31 \mathrm{G}$ and $\mathrm{HF} / 6$ $31+\mathrm{G} \mathrm{H}_{15}$ atom, at $\mathrm{HF} / 6-31 \mathrm{G}^{*} \mathrm{H}_{17}$ and at B3LYP/6-31G and B3PW91/6-31G $\mathrm{O}_{8}$ has the lowest shielding amount. In Molecule 3 in all of the methods, $N_{3}$ and $N_{5}$ atoms have the greatest positive shielding values. At HF/6-31G, HF/6$31 \mathrm{G}^{*}$ and $\mathrm{HF} / 6-31+\mathrm{G}, \mathrm{C}_{7}$ atom has the lowest shielding amount, also at B3LYP/6-31G, BLYP/6-31G and B3PW91/6-31G $\mathrm{O}_{9}$ has negative shielding value. In Molecule 4 , at all of the methods, $N_{5}$ has the negative shielding value, also $\mathrm{N}_{3}$ atom has negative value. In Molecule 5, at all of the methods, except HF/6-31G* level, $\mathrm{N}_{5}$ has the negative shielding value. At HF/6-31G, $N_{5}$ has positive shielding value.

Figure 1 shows the formula structures and the energy minimized 3-D chemical structures of 5-acetyl-6methylpyrimidine-2,4(1H,3H)-dione (molecule 1) , 3,4dihydro-5-phenylpyrimidin-2(1H)-one (Molecule 2), 1,2,3,4-tetrahydro-2-oxopyrimidine-5-carbaldehyde (Molecule 3), 1H-benzo[d]imidazole (Molecule 4) and 5,6dihydro-1H-benzo[d]imidazole-4,7-dione (Molecule using ball-and-stick model.

\section{REFERENCES}

AJafar A, Vijayakumar KN, Venkatraman BR, Venkatesh G (2009). Orbital-Electronic. J. Chem., 1: 306-309.

Andrzejewska M, Yepez-Mulia L, Tapia A, Cedillo-Rivera R, Laudy AE, Starosciak BJ, Kazimierczuk Z (2004). Synthesis, and antiprotozoal and antibacterial activities of S-substituted 4,6-dibromo- and 4,6dichloro-2-mercaptobenzimidazoles. Eur. J. Pharm. Sci., 21: 323329.

Ayhan-Kilcigil G, Kus C, Coban T, Can-Eke B, Iscan M (2004). Synthesis and Antioxidant Properties of Novel Benzimidazole Derivatives .J. Enzyme. Inhibitors Med. Chem., 19: 129-135.

Becke AD (1988). Density-Functional Exchange-Energy Approximation with Correct Asymptotic- Behavior. Phys. Rev. A., 38: 3098-3100.

Becke AD (1993). Density-Functional Thermochemistry 3. The Role of Exact Exchange. J. Chem. Phys., 98: 5648-5652.

Black E, Breed J, Breeze AL, Embrey K, Garcia R, Gero TW, Godfrey L, Kenny PW, Morley AD, Minshull CA, Pannifer AD, Read J, Rees A, Russell DJ, Toader D, Tucher J (2005). Structure-based design of protein tyrosine phosphatase-1B inhibitors. Bioorg. Med. Chem. Lett., 15: 2503.

Denny WA, Rewcastle GW, Bagley BC (1990). Potential antitumor agents. 59. Structure-activity relationships for 2phenylbenzimidazole-4-carboxamides, a new class of minimal DNAintercalating agents which may not act via topoisomerase II. J. Med. Chem., 33: 814

Dolle RE, Nelson KH (1999). Comprehensive Survey of Combinatorial Library Synthesis. J. Comb. Chem., 1: 235-282. b) Dolle RE (2000). Comprehensive Survey of Combinatorial Library Synthesis. J. Comb. Chem., 2: 383-433. c) Dolle RE (2004). Comprehensive Survey of Combinatorial Library Synthesis. J. Comb. Chem., 6: 623-679.

Fox DJ, Reckless J, Lingard H, Warren S, Grainger DJ (2009). Highly Potent, Orally Available Anti-inflammatory Broad-Spectrum Chemokine Inhibitors. J. Med. Chem., 52: 3591-3595.

Gardiner JM, Loyns CR, Burke A, Khan A, Mahmood N (2003). Design, 
synthesis and biological evaluation of benzimidazole/benzothiazole and benzoxazole derivatives as cyclooxygenase inhibitors. Bioorg. Med. Chem. Lett., 13: 657-660.

Ghassamipour S, Sardarian AR (2009). Friedländer synthesis of polysubstituted quinolines in the presence of dodecylphosphonic acid (DPA) as a highly efficient, recyclable and novel catalyst in aqueous media and solvent-free conditions. Tetrahedron Lett., 50: 514-519.

Gupta JK, Chaudhary A, Dudhe R, Varuna K, Sharma PK, Verma PK (2010). A review on the synthesis and therapeutic potential of pyrimidine derivatives. IJPSR., 1: 34-49.

Hazelton JC, Iddon B, Suschitzky H, Woolley LH (1995). 2Hbenzimidazoles (isobenzimidazoles). Part 10. Synthesis of polysubstituted o-phenylenediamines and their conversion into heterocycles, particularly 2-substituted benzimidazoles with known or potential anthelminthic activity . Tetrahedron., 51: 10771- 10794.

Islam I, Skibo EB, Dorr RT, Alberts DS (1991). Structure-activity studies of antitumor agents based on pyrrolo[1,2-a]benzimidazoles: new reductive alkylating DNA cleaving agents. J. Med. Chem., 34: 29542961.

Jung ME, Zimmerman CN, Lowen GT, Khan SI (1993). Diastereocontrol in intermolecular diels-alder reactions of allenic lactones: Synthetic approach to the plaunols . Tetrahedron Lett., 34: 4453-4456.

Karen K (2006). Antiviral drugs for cytomegalovirus diseases. Antiviral Res., 71: 154-163.

Kappe CO (1993). 100 years of the biginelli dihydropyrimidine synthesis. Terahedron., 49: 6937-6963.

KILCIG GA, Altanlar N (2006). Synthesis and Antifungal Properties of Some Benzimidazole Derivatives. Turk. J. Chem., 30: 223-228.

Kruse LL, Ladd DL, Harrsch PB, McCabe FL, Mong SM, Faucette L, Johnson R (1989). Synthesis, tubulin binding, antineoplastic evaluation, and structure-activity relationship of oncodazole analogs. J. Med. Chem., 32: 409-417.

Kuchkguzel I, Kuchkguzel G, Rollas S, Kiraz M (2001). Some 3thioxo/alkylthio-1,2,4-triazoles with a substituted thiourea moiety as possible antimycobacterials. Bioorg. Med. Chem. Lett., 11: 1703.

Kus C, Aylan-Kicigil G, Iscan M (2004). Synthesis and Antioxidant Properties of Some Novel Benzimidazole Derivatives on Lipid Peroxidation in the Rat Liver. Arch. Phar. Res., 27: 156-163.

Lee CT, Yang WT, Parr RG (1988). Development of the Colle-Salvetti Correlation-Energy Formula into a Functional of the Electron-Density. Phys. Rev. B., 37(2): 785-789.

Marsault E, Peterson ML (2011) Macrocycles Are Great Cycles: Applications, Opportunities, and Challenges of Synthetic Macrocycles in Drug Discovery. J. Med. Chem., 54 : 1961-2004

Mollaamin F, Layali I, Ilkhani AR, Monajjemi M (2010) Nanomolecular simulation of the voltage-gated potassium channel protein by gyration radius study . Afri J. Microbiol. Res., 4: 2795-2803.

Mollaamin F, Shahani poor K, Nejadsattari T, Monajjemi M (2010). Bionanomodeling of active site in oxidized azurin using by computational methods. Afr. J. Microbiol. Res., 4: 2098-2108.

Moller C, Plesset MS (1934). Note on an Approximation Treatment for Many-Electron Systems. Phys. Rev., 46: 618-622.

Monajiemi M, Afsharnezhad S, Jaafari MR, Abdolahi T, Nikosade A, Monajemi $H$ (2007). NMR shielding and a thermodynamic study of effect of environmental exposure to petrochemical solvent on DPPC, an important component of lung surfactant. Russ. J. Phys. Chem. A., 81: 1956-1963.

Monajjemi M, Azad MT, Haeri HH, Zare K, Hmedani Sh (2003). Ab initio conformational analysis of glutamic acid, chemical shift anisotropy and population studies. J. Chem. Res., 8: 454-456.

Monajjemi M, Heshmata M, Haeria HH (2006). QM/MM model study on properties and structure of some antibiotics in gas phase: comparison of energy and NMR chemical shift. Biochem. (Moscow), 71: 113-122.

Monajiemi M, Heshmat $\mathrm{H}$, Haeri HH, Keveh F (2006). Theoretical study of vitamin properties from combined QM/MM methods: comparison of chemical shifts and energy. Russ. J. Phys. Chem., 80: 1061-1068.

Monajjemi M, Honaparvar B, Khalili Hadad B, Ilkhani AR, Mollaamin F (2010). Thermo-chemical investigation and NBO analysis of some anxileotic as Nano- drugs. Afr. J. Pharm. Pharmacol., 4: 521-529.

Monajjemi M, Naderi F, Aghaie H, Yari M, Mansoor DN, Afsharnezhad S, Mollaamin F (2007). Study of thermodynamic functions value for CTAB. Res. J. Chem. Environ., 11: 56-62.
Monajjemi M, Rajaeian E, Mollaamin F, Naderi F, Saki S (2008). Investigation of NMR shielding tensors in 1,3 dipolar cycloadditions: solvents dielectric effect. Phys. Chem. Liqu., 46: 299-306.

Mollaamin F, Varmaghani Z and Monajjemi M (2011). Dielectric effect on thermodynamic properties in vinblastine by DFT/Onsager modeling.Phy. Chem. Liquids, 49: 318-336.

Nakano $\mathrm{H}$, Inoue $\mathrm{T}$, Kawasaki $\mathrm{N}$, Miyataka $\mathrm{H}$, Matsumoto $\mathrm{H}$, Taguchi $\mathrm{T}$, Inagaki N, Nagai H, Satoh T (1999). Synthesis of Benzimidazole Derivatives as Antiallergic Agents with 5-Lipoxygenase Inhibiting Action. Chem. Pharm. Bull., 47: 1573-1578.

Ozden S, Atabey D, Yildiz S, Goker H (2005). Synthesis and potent antimicrobial activity of some novel methyl or ethyl $1 \mathrm{H}-$ benzimidazole-5-carboxylates derivatives carrying amide or amidine groups. Bioorg. Med. Chem., 13: 1587-1597.

$\mathrm{Pal}$ K, Pore S, Sinha S, Janardhanan R, Mukhopadhyay D, and Banerjee R (2011). Structure-Activity Study To Develop Cationic Lipid-Conjugated Haloperidol Derivatives as a New Class of Anticancer Therapeutics. J. Med. Chem., 14: 2378-2390.

Porcari AR, Devivar RV, Kucera LS, Drach JC, Townsend LB (1998). Design, Synthesis, and Antiviral Evaluations of 1-(Substituted benzyl)-2-substituted-5,6-dichlorobenzimidazoles as Nonnucleoside Analogues of 2,5,6-Trichloro-1-( $\beta$-D-ribofuranosyl)benzimidazole. J. Med. Chem., 41: 1252-1262.

Predew JP, Wang Y (1992). Accurate and simple analytic representation of the electron-gas correlation energy. Phys. Rev. B., 45: 13244-13249.

Ramla MM, Omar MA, Tokuda H, El.Diwani HI (2007). Synthesis and inhibitory activity of new benzimidazole derivatives against Burkitt's lymphoma promotion. Bioorg. Med. Chem., 15: 6489-6496.

Rosowsky A, Vaidya CM, Bader H, Wright JE, Teicher BA (1997) Analogues of $\mathrm{N}^{\alpha}$-(4-Amino-4-deoxypteroyl)- $\mathrm{N}^{\delta}$-hemiphthaloyl-Lornithine (PT523) Modified in the Side Chain: Synthesis and Biological Evaluation. J. Med. Chem., 40: 286-299.

Samia R, Soda A, El-Hesham M, Fahmy TY (2006). Synthesis of Novel Benzofuran and Related Benzimidazole Derivatives for Evaluation of In Vitro Anti-HIV-1, Anticancer and Antimicrobial Activities. Arch. Pharm. Res., 29: 826-833.

Senten K, Venken PVV, Meester ID, Lambeir AM, Scharpe S, Haemers A, Augustyns K (2003). Design, Synthesis, and SAR of Potent and Selective Dipeptide-Derived Inhibitors for Dipeptidyl Peptidases. J. Med. Chem., 46: 5005.

Smith JM, Gard J, Cummings W, Kanizsai A, Krchnak V (1999). Necklace-Coded Polymer-Supported Combinatorial Synthesis of 2 Arylaminobenzimidazoles. J. Comb. Chem., 1: 368-370.

Souness E, Aldous D, Sargent C (2000). Immunosuppressive and antiinflammatory effects of cyclic AMP phosphodiesterase (PDE) type 4 inhibitors. Immunopharmacol., 47: 127-162.

Spasov AA, Yozhitsa IN, Bugaeva LI, Anisimova VA (1999). Benzimidazole derivatives: Spectrum of pharmacological activity and toxicological properties. J. Pharm. Chem., 33: 232-243.

Sztanke K, Pasternak K, Sidor-Wojtowicz A, Truchlinska J, Jozwiak $\mathrm{K}(2006)$. Synthesis of imidazoline and imidazo[2,1-c][1,2,4]triazole aryl derivatives containing the methylthio group as possible antibacterial agents. Bioorg. Med. Chem., 14: 3635-3642.

Sun H, Liu L, Lu J, Bai L, Li X, Nikolovska-Coleska Z, McEachern D, Yang CY, Qiu S, Yi H, Sun D, Wang S (2011). Potent Bivalent Smac Mimetics: Effect of the Linker on Binding to Inhibitor of Apoptosis Proteins (IAPs) and Anticancer Activity. J. Med. Chem., 12: 33063318.

Tatsuta M, Kataoka M, Yasoshima K, Sakakibara S, Shimazaki M, Yura T, Li Y, Yamamoto N, Gupta J, Urbahns K (2005). Benzimidazoles as non-peptide luteinizing hormone-releasing hormone (LHRH) antagonists. Part 3: Discovery of 1-(1H-benzimidazol-5-yl)-3-tertbutylurea derivatives. Bioorg. Med. Chem. Lett., 15: 2265-2269.

Vijayakumar K, and Jafar Ahamed A (2010). Synthesis, Anti-Tumor, Anti-Diabetic, and Anti- Asthmatic Activitives of Some Novel Benzimidazole Derivatives . J. Chem. Pharm. Res., 2: 215-224.

Wu Z, Ede NJ, Mathieu MN (2003). Solid-phase synthesis of benzimidazole $\mathrm{N}$-oxides on SynPhase ${ }^{\mathrm{TM}}$ Lanterns. Tetrahedron Letters., 44 : 2293-2296.

Yadav JS, Srivastava YK (2011). A facile synthesis and antimicrobial activity of some new 2-subsituted benzimidazole derivatives carrying 
pyridine. Der Chemica Sinica., 2: 1-7.

Young WB, Sprengeler P, Shrader WD, Li Y, Rai R, Verner E, Jenkins

T, Fatheree P, Kolesnikov A, Janc JW, Cregar L, Elrod K, Katz B

(2006). Generation of potent coagulation protease inhibitors utilizing

zinc-mediated chelation. Bioorg. Med. Chem. Lett., 16: 710-713. 